UC-2000

Issued: October 1997

Correction to the MCNPTM

Perturbation Feature for

Cross-Section Dependent Tallies

J. D. Densmore

G. W. McKinney

J. S. Hendricks 


\section{DISCLAmER}

Portions of this docoment may be illegible in electronic image products. Imsges are produced from the best available original documenter. 


\section{DISCLAIMER}

This report was prepared as an account of work sponsored by an agency of the Uinited States Government. Neither the United States Government nor any agency thereof, nor any of their employees, make any warranty, express or implied, or assumes any legal liability or responsibility for the accuracy, completeness, or usefulness of any information, apparatus, product, or process disclosed, or represents that its use would not infringe privately owned rights. Reference herein to any specific commercial product, proces, or service by trade name, trademark, manufacturer, or otherwise does not necessarily constitute or imply its endorsement, recommendation, or favoring by the United States Government or any agency thereof. The views and opinions of authors expressed herein do not necessar. ily state or reflect those of the United States Government or any agency thereof. 


\title{
CORRECTION TO THE MCNPTM PERTURBATION FEATURE FOR CROSS-SECTION DEPENDENT TALLIES
}

\author{
by
}

\author{
J. D. Densmore, G. W. McKinney, and J. S. Hendricks
}

\begin{abstract}
The differential operator perturbation technique is a new feature of the Monte Carlo N-Particle Transport Code MCNP' version 4B that will allow users to calculate the effects of cross-section data perturbations on tallies. The implementation of the differential operator perturbation technique in MCNP assumes that the tally is independent of any perturbed cross-section data, an assumption that may not be valid for some tallies. We provide derivations of both the first- and second-order corrected perturbations. In addition, the appropriate perturbation corrections are demonstrated so users may accurately calculate perturbation effects for any cross-section dependent tally. Finally, corrected perturbations from six example problems are compared to actual MCNP results.
\end{abstract}

MCNP is a trademark of the Regents of the University of California, Los Alamos National Laboratory. 


\section{INTRODUCTION}

Perturbation techniques are powerful tools for radiation transport problems. These techniques allow the user to determine the effects of small changes in the problem. For many years the users of the Monte Carlo code MCNP have expressed interest in adding a perturbation feature to MCNP. The recently released MCNP4B has a new feature that uses the differential operator technique to calculate the effects of perturbations of cross-section data. The perturbations of cross-section data can include density and composition changes for specific reactions over specific energy ranges. The perturbation feature is used to calculate the effects of cross-section changes on tallies.

The differential operator technique allows the precise calculation of perturbations even if the uncertainty in the unperturbed tally is greater than the calculated perturbation. The differential operator technique is approximated by a second-order Taylor series expansion. The implementation of this expansion assumes that the tally response estimator is independent of any perturbed cross-section data. For some tallies, such as reaction rate estimators, this assumption is invalid. To correct for this assumption, a correction term must be added to the calculated perturbation to account for the tally response estimator's dependence on the perturbed crosssection data.

This report will provide the correction to the first and second-order Taylor series expansion and will provide verification of this correction. In Section II we derive the corrected first-order perturbation by starting from the original Taylor series. In addition, the first- and second-order corrected perturbations are derived beginning with the coefficients as implemented in MCNP. In Section III, the use of this correction will be demonstrated for several types of problems, illustrating how perturbations of cross-section dependent tallies can be done with MCNP4B. Finally, in Section IV we compare perturbed calculations with actual tallies for various types of problems, including fixed-source reaction rate problems and track-length estimates for $\mathrm{k}_{\mathrm{eff}}$ to demonstrate the validity of the correction. 


\section{PERTURBATION CORRECTION}

\section{A. Theory}

We repeat here much of the theory presented in X-6:GWM-94-1242 and LA-13098. ${ }^{3}$ Consider a track-length estimate which is a function of some cross-section data set $\mathbf{X}$. A subset of this data set, $\mathbf{X}_{\mathbf{v}}$, can be defined as

$$
X_{v} \equiv\left\{x_{b}(h)=K_{b}(h) e^{\nu} ; b \in B ; h \in H\right\}
$$

where $K_{b}(h)$ is some constant, $B$ represents a set of macroscopic cross sections, and $H$ represents a set of energies. This definition allows for a subset of $\mathbf{X}$ to be represented by a single parameter v. Notice that

$$
\frac{d}{d v} x_{b}(h)=K_{b}(h) e^{\nu}=x_{b}(h)
$$

and

$$
d v=\frac{d x_{b}(h)}{x_{b}(h)}
$$

This equation shows that $d v$ is simply the fractional change in the macroscopic cross section.

For Monte Carlo calculations, a track-length estimate tally is given by

$$
c=\sum_{j} t_{j} q_{j}
$$

where $t_{j}$ is the tally response estimator of path segment $j$ and $q_{j}$ is the probability of path segment j. Converting Eq. (4) from a path segment estimator to a history-based estimator, ${ }^{2}$ the result is

$$
\langle c\rangle=\frac{1}{N} \sum_{i} \sum_{j^{\prime}} t_{j^{\prime}}
$$


where the summation over $\mathrm{j}^{\prime}$ includes only those path segments in particle history $\mathrm{i}$ and $\mathrm{N}$ is the total number of particle histories. Equation (5) gives the average value of the tally, which is the tally as reported by MCNP.

For cross-section dependent tallies, the tally response estimator is given by

$$
t_{j^{\prime}}=D x_{b}(h) \lambda_{j^{\prime}}
$$

where $x_{b}(h)$ is the cross section upon which the tally response estimator is dependent, $D$ is other data upon which the tally response estimator is dependent, and $\lambda_{j^{\prime}}$ is the length of the last track in path segment $j^{\prime}$. For example, consider a track-length estimate for $\mathrm{k}_{\text {eff }}$ which is given by

$$
k_{\text {eff }}=\rho \iint_{V E} v(E) \sigma_{f}(E) \phi(\not, E) d V d E
$$

This equation can be calculated with the use of track-length estimate of flux and a tally multiplier card. In this case, the cross section is

$$
x_{b}(h)=\rho \sigma_{f}(h)=\Sigma_{f}(h)
$$

and the other data is

$$
D=v(h) / V
$$

Note that the track-length estimate of the flux for path segment $j^{\prime}$ is

$$
\phi=\frac{\lambda_{j^{\prime}}}{V}
$$

For other track-length estimates, the results are similar.

As stated before, MCNP uses a second-order Taylor series to calculate perturbations. This series takes the form

$$
\Delta c=\frac{d c}{d v} \Delta v+\frac{d^{2} c}{d v^{2}} \Delta v^{2}
$$


which can be rewritten as

$$
\Delta c=u_{1} \Delta v+u_{2} \Delta v^{2}
$$

where $\Delta \mathrm{c}$ is the change in the tally caused by the perturbation, $\mathrm{u}_{1}$ and $\mathrm{u}_{2}$ are coefficients of the series, and $\Delta v$ is the percent change in the data (density, concentration, etc.). The $\mathrm{n}^{\text {th }}$ order coefficient is given by

$$
u_{n}=\frac{1}{n !} \cdot \frac{d^{n} c}{d v^{n}}
$$

which can be written as

$$
u_{n}=\frac{1}{n !} \sum_{b \in B} \sum_{B \in H} x_{b}^{n}(h)\left(\frac{\partial^{n} c}{\partial x_{b}^{n}(h)}\right)
$$

where $B$ represents a set of macroscopic cross sections and $\mathrm{H}$ represents a set of energies or an energy interval. Using Eq. (4), this becomes

$$
u_{n}=\frac{1}{n !} \sum_{j}\left[\sum_{b \in B} \sum_{h \in H} x_{b}^{n}(h)\left(\frac{\partial^{n}}{\partial x_{b}^{n}(h)}\left(t_{j} q_{j}\right)\right)\right]
$$

or

$$
u_{n}=\frac{1}{n !} \sum_{j} \gamma_{n j} t_{j} q_{j}
$$

where

$$
\gamma_{n j} \equiv \sum_{b \in B} \sum_{h \in H} x_{b}^{n}(h)\left(\frac{\partial^{n}}{\partial x_{b}^{n}(h)}\left(t_{j} q_{j}\right)\right)\left(\frac{1}{t_{j} q_{j}}\right)
$$

With some manipulations presented in Ref. 2, the path segment estimator of Eq. (16) can be converted to a particle history estimator of the form 


$$
u_{n}=\sum_{i} V_{n i} p_{i}
$$

where $\mathrm{p}_{\mathrm{i}}$ is the probability of the $\mathrm{i}^{\text {th }}$ history and $\mathrm{V}_{\mathrm{ni}}$ is the $\mathrm{n}^{\text {th }}$ order coefficient estimator for history $i$, given by

$$
V_{n i} \equiv \frac{1}{n !} \sum_{j^{\prime}} \gamma_{n j^{\prime}} t_{j^{\prime}}
$$

Note that this sum involves only those path segments $j^{\prime}$ in particle history i. Equation (19) shows how the history estimator for the $\mathrm{n}^{\text {th }}$ order coefficient can be computed from the track (or path segment) based operator $\gamma_{n j^{\prime}}$. The Monte Carlo expected value of $u_{n}$ becomes

$$
\begin{aligned}
\left\langle u_{n}\right\rangle & =\frac{1}{N} \sum_{i} V_{n i} \\
& =\frac{1}{N n !} \sum_{i}\left(\sum_{j^{\prime}} \gamma_{n j^{\prime}} t_{j^{\prime}}\right),
\end{aligned}
$$

for a sample of $\mathrm{N}$ particle histories.

For a first-order perturbation, the differential operator becomes

$$
\gamma_{1 j^{\prime}}=\sum_{b \in B} \sum_{h \in H} x_{b}(h)\left(\frac{1}{q_{j^{\prime}}}\right)\left(\frac{\partial q_{j^{\prime}}}{\partial x_{b}(h)}\right)
$$

assuming the response estimator $t_{j^{\prime}}$ is not a function of $x_{b}(h)$. The path segment probability can be written as the product of track probabilities

$$
q_{j^{\prime}}=\prod_{k=0}^{m} r_{k}
$$

where $r_{k}$ is the probability of track $k$ and segment $j^{\prime}$ contains $m+1$ tracks. In terms of tracks, the operator becomes 


$$
\gamma_{1 j^{\prime}}=\sum_{k=0}^{m}\left[\sum_{b \in B} \sum_{h \in H} x_{b}(h)\left(\frac{1}{r_{k}}\right)\left(\frac{\partial r_{k}}{\partial x_{b}(h)}\right)\right]
$$

or

$$
\gamma_{1 j^{\prime}}=\sum_{k=0}^{m} \beta_{j^{\prime} k}
$$

where

$$
\beta_{j^{\prime} k} \equiv \sum_{b \in B} \sum_{h \in H} x_{b}(h)\left(\frac{1}{r_{k}}\right)\left(\frac{\partial r_{k}}{\partial x_{b}(h)}\right)
$$

Defining track probabilities, $\mathrm{r}_{\mathrm{k}}$, in terms of Monte Carlo transport parameters is the final step of this derivation. If the $\mathrm{k}^{\text {th }}$ track starts with a neutron undergoing reaction type "a" at energy $E^{\prime}$ and is scattered from angle $\theta^{\prime}$ to angle $\theta$, and energy $E$ continues for a length $\lambda_{k}$ and collides, then

$$
r_{k}=\left(\frac{x_{a}\left(E^{\prime}\right)}{x_{T}\left(E^{\prime}\right)}\right) P_{a}\left(E^{\prime} \rightarrow E ; \theta^{\prime} \rightarrow \theta\right) d E d \theta\left(e^{-x_{T}(E) \lambda_{k}}\right) x_{T}(E) d \lambda
$$

where $x_{a}\left(E^{\prime}\right)$ is the macroscopic reaction cross section at energy $E^{\prime}, x_{T}\left(E^{\prime}\right)$ is the total cross section at energy $E^{\prime}$, and $P_{a}\left(E^{\prime}->E ; \theta^{\prime}->\theta\right) d E d \theta$ is the probability distribution function in phase space of the emerging neutron. Equation (25) becomes

$$
\beta_{j k}=\sum_{b \in B h \in H} \sum_{h E^{\prime}}\left(\delta_{b a}-\frac{\delta_{h E} x_{b}\left(E^{\prime}\right)}{x_{T}\left(E^{\prime}\right)}-\delta_{h E} x_{b}(E) \lambda_{k}+\frac{\delta_{h E^{x}} x_{b}(E)}{x_{T}(E)}\right),
$$

where $\delta_{\mathrm{hE}}$ and $\delta_{\mathrm{ba}}$ are unity if $\mathrm{h}=\mathrm{E}$ and $\mathrm{b}=\mathrm{a}$; otherwise, they vanish. A similar derivation can be performed for other types of tracks (i.e., collision to boundary, boundary to collision, and boundary to boundary), leading to one or more of these four terms. Finally, combining Eqs. (20) and (24) gives the expected value of the first-order coefficient 


$$
\left\langle u_{1}\right\rangle=\frac{1}{N} \sum_{i}\left[\sum_{j^{\prime}}\left(\sum_{k=0}^{m} \beta_{j^{\prime} k}\right) t_{j^{\prime}}\right]
$$

where $\beta_{j^{\prime} k}$ is calculated from one or more terms of Eq. (27) for track k.

For a second-order perturbation, the differential operator becomes

$$
\gamma_{2 j}=\sum_{b \in B} \sum_{h \in H} x_{b}^{2}(h)\left(\frac{1}{q_{j}}\right)\left(\frac{\partial^{2} q_{j}}{\partial x_{b}^{2}(h)}\right)
$$

again assuming the response estimator $t_{j^{\prime}}$ is not a function of $x_{b}(h)$. Omitting steps presented in Ref. 2, the second-order operator becomes

$$
\gamma_{2, j^{\prime}}=\sum_{k=0}^{m}\left(\alpha_{j^{\prime} k}-\beta_{j^{\prime} k}^{2}\right)+\left(\sum_{k=0}^{m} \beta_{j^{\prime} k}\right)^{2},
$$

where

$$
\mathbf{0}_{j^{\prime} k}^{\prime} \equiv \sum_{b \in B} \sum_{h \in H} x_{b}^{2}(h)\left(\frac{1}{r_{k}}\right)\left(\frac{\partial^{2} r_{k}}{\partial x_{b}^{2}(h)}\right)
$$

It is evident that $\gamma_{2 j^{\prime}}$ requires little additional effort to $\gamma_{1 j^{\prime}}$, namely the computation of $\alpha_{j^{\prime} k}$. If $\mathrm{r}_{k}$ is given by Eq. (26), then $\alpha_{j^{\prime} k}$ becomes

$$
\begin{aligned}
\alpha_{j^{\prime} k}= & \sum_{b \in B} \sum_{h \in H} \frac{2 \delta_{h E^{*}} x_{b}^{2}\left(E^{\prime}\right)}{x_{T}^{2}\left(E^{\prime}\right)}-\frac{2 \delta_{h E^{\prime}} \delta_{b a} x_{b}\left(E^{\prime}\right)}{x_{T}\left(E^{\prime}\right)} \\
& +\delta_{h E} x_{b}^{2}(E) \lambda_{k}^{2}-\frac{\left.2 \delta_{h E^{x}} x_{b}^{2} E\right) \lambda_{k}}{x_{T}(E)} .
\end{aligned}
$$

Once again, for other types of tracks one or more of these four terms is required. The expected value of the second-order coefficient, via Eq. (20), becomes 


$$
\left\langle u_{2}\right\rangle=\frac{1}{2 N} \sum_{i}\left[\sum_{j}\left(\sum_{k=0}^{m}\left(\alpha_{j^{\prime} k}-\beta_{j^{\prime} k}^{2}\right)+\left(\sum_{k=0}^{m} \beta_{j^{\prime} k}\right)^{2}\right) t_{j}\right],
$$

where $\beta_{j^{\prime} k}$ is given by one or more terms of Eq. (27) and $\alpha_{j^{\prime} k}$ by one or more terms of Eq. (32) for track $\mathrm{k}$.

These coefficients were derived with the assumption that the tally response estimator is independent of any perturbed cross sections. If this assumption is not valid, a term must be added to these coefficients that accounts for the dependence of the tally response estimator on the perturbed cross section. This correction term is given in Appendix D of Ref. 3 as

$$
R_{1 j^{\prime}}=\sum_{b \in B} \sum_{h \in H} x_{b}(h)\left(\frac{1}{t_{j}}\right)\left(\frac{\partial t_{j^{\prime}}}{\partial x_{b}(h)}\right)
$$

where $\mathrm{R}_{1 \mathrm{j}}$, is the correction term, $\mathrm{B}$ refers to a set of macroscopic cross sections, and $\mathrm{H}$ refers to a set of energies. The corrected coefficients, also given in Appendix D of Ref. 3, are then

$$
u_{1}=\frac{1}{N} \sum_{i}\left[\sum_{j^{\prime}}\left(\sum_{k=0}^{m} \beta_{j^{\prime} k}+R_{1 j^{\prime}}\right) t_{j^{\prime}}\right]
$$

and

$$
u_{2}=\frac{1}{2 N} \sum_{i}\left[\sum_{j}\left(\sum_{k=0}^{m}\left(\alpha_{j^{\prime} k}-\beta_{j^{\prime} k}^{2}\right)+\left(\sum_{k=0}^{m} \beta_{j^{\prime} k}+R_{1 j}\right)^{2}-R_{1 j^{\prime}}^{2}\right) t^{\prime}\right]
$$

\section{B. First-Order Correction - Taylor Series}

Considering only the first term of the Taylor series and substituting in Eq. (4), Eq. (11) becomes

$$
\Delta c=\frac{d c}{d v} \Delta v=\frac{d}{d v}\left[\sum_{j} t_{j} q_{j}\right] \Delta v
$$

Differentiating Eq. (37) gives 


$$
\Delta c=\left[\sum_{j} t \frac{d q_{j}}{j d v}+q_{j} \frac{d t}{d v}\right] \Delta v
$$

The original Taylor series was derived assuming that the response estimator was independent of any perturbed data. In such cases, the derivative of $t_{j}$ with respect to $v$ is equal to zero, and the uncorrected perturbation is

$$
\Delta c=\left[\sum_{j} t \frac{d q_{j}}{d v}\right] \Delta v=P E R T u c 1
$$

where PERTuc1 is the uncorrected first-order perturbation as reported by MCNP. Substituting Eq. (39) into Eq. (38) gives

$$
\Delta c=P E R T u c 1+\left[\sum_{j} q_{j} \frac{d t}{d v}\right] \Delta v
$$

Substituting Eq. (6) into Eq. (40) and assuming the track length is not a function of the perturbed data, one obtains

$$
\Delta c=P E R T u c 1+\left[\sum_{j} q_{j} D \lambda_{j} \frac{d}{d v} x_{b}(h)\right] \Delta v
$$

Using the results from Eq. (2), the result is

$$
\Delta c=P E R T u c 1+\left[\sum_{j} q_{j} D \lambda_{j} x_{b}(h)\right] \Delta v=P E R T u c 1+\sum_{j} q_{j} t_{j} \Delta v
$$

Using the Monte Carlo definition of a tally, Eq. (4), the expected value of the first-order corrected perturbation is

$$
\langle\Delta c\rangle=P E R T u c 1+\mathrm{TALLY} \cdot \Delta v
$$

which is the uncorrected perturbation plus the unperturbed tally times the percent change in the perturbed data. 


\section{First-Order Correction - Coefficients}

Considering only the first term and substituting in Eq. (35), the expected value of Eq. (12) becomes a

$$
\langle\Delta c\rangle=u_{1} \Delta v=\frac{1}{N} \sum_{i}\left[\sum_{j^{\prime}}\left(\sum_{k=0}^{m} \beta_{j^{\prime} k}+R_{1 j^{\prime}}\right)^{j^{\prime}}\right] \Delta v .
$$

With a little algebraic manipulation, one obtains

$$
\langle\Delta c\rangle=\frac{1}{N} \sum_{i}\left[\sum_{j^{\prime}}\left(\sum_{k=0}^{m} \beta_{j^{\prime} k^{\prime}}+R_{1 j^{\prime}} t_{j}\right)\right] \Delta v .
$$

Separating the two terms, Eq. (45) becomes

$$
\langle\Delta c\rangle=\frac{1}{N} \sum_{i}\left[\sum_{j^{\prime}} \sum_{k=0}^{m} \beta_{j^{\prime} k^{\prime}} t_{j^{\prime}}\right] \Delta v+\frac{1}{N} \sum_{i}\left[\sum_{j^{\prime}} R_{1 j^{\prime}} t^{j^{\prime}}\right] \Delta v .
$$

Notice that the coefficient of the first term of Eq. (46) is identical to Eq. (28), the uncorrected firstorder perturbation. Thus, the corrected first-order perturbation is simply the uncorrected firstorder perturbation plus some other term.

Now, let's examine the $R_{1 j^{\prime}}$ term. By substituting Eq. (6) into Eq. (34), the result is

$$
R_{1 j^{\prime}}=\sum_{b \in B h \in H} \sum_{b} x_{b}(h)\left(\frac{1}{D x_{b}(h) \lambda_{j^{\prime}}}\right)\left(\frac{\partial}{\partial x_{b}(h)}\left[D x_{b}(h) \lambda_{j^{\prime}}\right]\right) .
$$

Performing the derivative and simplifying, Eq. (47) becomes

$$
R_{1 j^{\prime}}=\sum_{b \in B} \sum_{h \in H} \frac{x_{b}(h) D \lambda_{j^{\prime}}}{D x_{b}(h) \lambda_{j^{\prime}}}=1
$$

Equation (48) holds only if all cross sections involved in the tally (i.e., on the MCNP FM card) are perturbed by the same amount over all energy ranges. Special perturbations, such as concentration perturbations or the use of the RXN or ERG keywords on the FM card, will cause 
$R_{1 j^{\prime}}$ to be less than unity. This difficulty can be solved by separating the tally into bins that are perturbed by the same amourt.

Now Eq. (48) can be substituted into Eq. (46), giving

$$
\langle\Delta c\rangle=\operatorname{PERTuc1}+\left[\frac{1}{N} \sum_{i} \sum_{j^{\prime}} t_{j^{\prime}}\right] \Delta \mathrm{v}
$$

where PERTuc1 is the uncorsected first-order perturbation as reported by MCNP. Notice that the second term of Eq. (49) is identical to Eq. (5), the history-based estimate of a tally. Thus, Eq. (49) can be written as

$$
\langle\Delta c\rangle=P E R T u c 1+T A L L Y \cdot \Delta v
$$

where TALLY is the unperturbed tally as reported by MCNP. Note that this equation is consistent with Eq. (48) and therefore Eq. (50) gives the corrected first-order perturbation for cross-section dependent tallies.

\section{Second-Order Correction - Coefficients}

Now let's consider only the second term of Eq. (12). Substituting Eq. (36) and setting $R_{1 j^{\prime}}$ equal to one, the expected value of Eq. (12) becomes

$$
\langle\Delta c\rangle=\frac{1}{2 N} \sum_{i}\left[\sum_{j}\left(\sum_{k=0}^{m}\left(\alpha_{j^{\prime} k}-\beta_{j^{\prime} k}^{2}\right)+\left(\sum_{k=0}^{m} \beta_{j^{\prime} k}+1\right)^{2}-1\right) t_{j}\right] \Delta v .
$$

Performing a little algebraic manipulation on Eq. (51) gives

$$
\langle\Delta c\rangle=\frac{1}{2 N} \sum_{i}\left[\sum_{j^{\prime}}\left(\sum_{k=0}^{m}\left(\alpha_{j^{\prime} k}-\beta_{j^{\prime} k}^{2}\right)+\left(\sum_{k=0}^{m} \beta_{j^{\prime} k}\right)^{2}+2 \sum_{k=0}^{m} \beta_{j^{\prime} k}+1-1\right) t_{j^{\prime}}\right] \Delta v
$$

Again, one can manipulate the corrected perturbation so that it becomes the uncorrected perturbation plus another term. In this case Eq. (52) becomes 


$$
\langle\Delta c\rangle=\frac{1}{2} \sum_{i}\left[\sum_{j^{\prime}}\left(\sum_{k=0}^{m}\left(\alpha_{j^{\prime} k}-\beta_{j^{\prime} k}^{2}\right)+\left(\sum_{k=0}^{m} \beta_{j^{\prime} k}\right)^{2}\right) t_{j^{\prime}}\right] \Delta v+\frac{1}{N} \sum_{i}\left[\sum_{j^{\prime}}\left(\sum_{k=0}^{m} \beta_{j^{\prime} k}\right) t_{j^{\prime}}\right] \Delta v
$$

Notice that the coefficient of the first term in Eq. (53) is identical to Eq. (33), the uncorrected second-order perturbation, and the coefficient of the second term is identical to Eq. (28), the uncorrected first-order perturbation. Equation (53) then becomes

$$
\langle\Delta c\rangle=P E R T u c 2+P E R T u c 1 \cdot \Delta v
$$

where PERTuc2 is the uncorrected second-order perturbation as reported by MCNP. Adding Eq. (54) to Eq. (50), the total corrected perturbation becomes

$$
\langle\Delta c\rangle=P E R T u c T+T A L L Y \cdot \Delta v+P E R T u c 1 \cdot \Delta v
$$

where PERTucT is the total perturbation as reported by MCNP.

\section{USE OF THE CORRECTION}

The following section will demonstrate the use of the perturbation correction for both density and composition changes.

\section{A. Density Perturbations}

For density perturbations, the implementation of Eq. (55) is elementary. The unperturbed tally is printed in the output, and the total and first-order uncorrected perturbations can be obtained by the use of a method $=1$ and a method $=2$ keyword in the PERT card, respectively. The percent change in the cross section will be just the percent change in the density.

The following example is a density perturbation of the Lady Godiva critical assembly, one of the problems included in the next section. In this example, the perturbation in $\mathbf{k}_{\text {eff }}$ caused by a density change from $18.74 \mathrm{~g} / \mathrm{cc}$ to $20.0 \mathrm{~g} / \mathrm{cc}$ will be calculated. In addition to the normal input deck, the following cards were added: 


\section{f $4: \mathrm{n} 1$}

sd4 1

$$
\begin{aligned}
& \text { fm4 }(-11-6-7) \\
& \text { Pert } 1: \text { cell }=1 \text { rho }=-20 \text { method }=2 \\
& \text { Pert } 2: \text { cell }=1 \text { rho= }=-20 \text { method }=1
\end{aligned}
$$

The first card calculates the track-length estimate of the flux in cell 1 (the fissile cell). The second card sets the volume of the cell equal to unity so that a track-length estimate of the neutron population is calculated. The third card multiplies the tally by the atom density, fission nu, and fission cross section of material 1 . These three cards will calculate $k_{\text {eff }}$. The last two cards perturb the density of cell one to $20.0 \mathrm{~g} / \mathrm{cc}$ and calculate the change in the tally using the firstorder perturbation (method=2) and the total perturbation (method=1). The following output was generated by this input (erross given are relative errors):

$$
\begin{gathered}
\text { TALLY }=0.998398 \pm 0.0008 \\
\text { PERTuc1 }=-1.31884 \mathrm{E}-2 \pm .0114 \\
\text { PERTucT }=-1.35007 \mathrm{E}-2 \pm .0107
\end{gathered}
$$

Using Eq. (55), the corrected perturbation is

$$
\begin{aligned}
\langle\Delta c\rangle & =-0.0135007-0.0131884\left(\frac{20-18.74}{18.74}\right)+0.998398\left(\frac{20-18.74}{18.74}\right) \\
& =0.05274 \pm 0.00015,
\end{aligned}
$$

where the errors given are standard deviations. Notice that the corrected perturbation is positive, and the uncorrected perturbation is negative. If an uncorrected perturbation is used in this problem, not only will an incorrect value for $k_{\text {eff }}$ be calculated, but the user will believe that an increase in density will cause $k_{\text {eff }}$ to decrease, which is obviously incorrect.

\section{B. Composition Perturbation}

Composition perturbations are a bit more complicated. Since the cross section of each isotope is being perturbed separately, the tally must be broken up into a bin for each isotope. The multiplier for the bin will be the macroscopic cross section of interest for that particular isotope, and the percent change in the macroscopic cross section will be the percent change in the weight 
or atom fraction. In this manner each individual bin can be corrected and then added together to obtain the total corrected perturbation.

The following example is a composition perturbation of the Lady Godiva critical assembly, one of the problems included in the next section. In this problem the perturbation in $\mathrm{k}_{\text {eff }}$ caused by a composition change in the fissile material is calculated. The original composition was $94.73 \% \mathrm{U}-2355.27 \% \mathrm{U}-238$, and the new composition is $87 \% \mathrm{U}-235,13 \% \mathrm{U}-238$. The percents given are weight percents. In addition to the normal input deck, the following cards are needed.

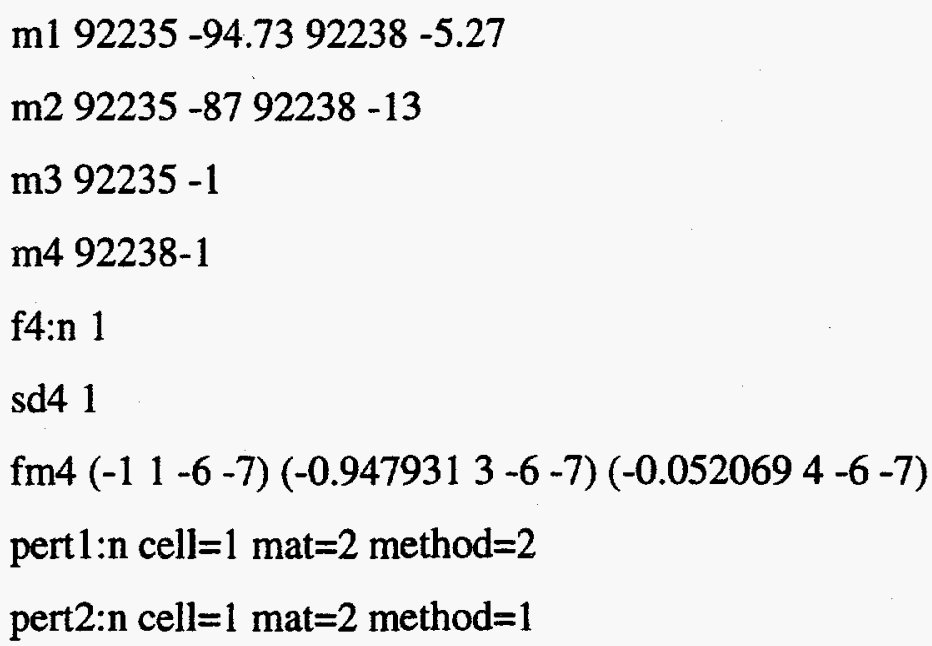

The first material card is the original composition, whereas the second material card is the perturbed composition. The last two material cards will be used to divide the tally into a separate bin for each isotope. The tally cards are identical to the last example, except that two new bins have been added to the FM card. These multiply the flux by each isotope's respective macroscopic cross section (the first number is atom fraction of that isotope in the material) so that each bin can be corrected separately. The PERT cards change the composition of cell one from material one to material two and calculate the change in $\mathrm{k}_{\text {eff }}$ by using the first order and total perturbation. The following output was generated (errors given are relative errors):

\footnotetext{
$\operatorname{TALLY}(235)=0.988752 \pm 0.0008$

$\operatorname{TALLY}(238)=0.00964622 \pm 0.0017$

PERTuc1 $(235)=0.0264468 \pm 0.0264$

PERTuc1 $(238)=0.000059659 \pm 0.0961$

PERTucT $=0.0256058 \pm 0.0318$
} 
where the (235) and (238) refer the to U-235 and U-238 bins of the tally. Using the last two terms of Eq. (55), the correction to the $\mathrm{U}-235$ bin is

$$
\begin{aligned}
\langle\Delta c\rangle^{235} & =0.988752\left(\frac{87-94.73}{94.73}\right)+0.0264468\left(\frac{87-94.73}{94.73}\right) \\
& =-0.082841 \pm 0.000086 .
\end{aligned}
$$

The correction to the $\mathrm{U}-238$ bin is

$$
\begin{aligned}
\langle\Delta c\rangle^{238} & =0.00964622\left(\frac{13-5.27}{5.27}\right)+0.5966 \times 10^{-4}\left(\frac{13-5.27}{5.27}\right) \\
& =0.014237 \pm 0.000025
\end{aligned}
$$

And the total corrected perturbation is

$$
\begin{aligned}
\langle\Delta c\rangle & =0.0256058+0.014237-0.082841 \\
& =-0.04300 \pm 0.00082
\end{aligned}
$$

where the errors given are standard deviations.

\section{VERIFICATION RIESULTS}

In this section, corrected perturbations are compared to actual MCNP results. Six test problems were used that will demonstrate the validity of density and composition perturbations for criticality and fixed neutron and gamma sources. For the most part, these problems were taken from the MCNP4B test suite except that two problems involving the Lady Godiva critical assembly were included because of the lack of appropriate criticality problems in the test suite. The problems taken from the test suite have been modified to emphasize the use of a perturbed cross-section dependent tally. In some cases, tally, variance reduction, and physics cards have been removed or altered. However, the original geometry and materials remain the same. This 
verification differs from a benchmark in that results calculated using the MCNP perturbation feature will be compared to actual MCNP results, not experimental results.

Each example consists of five input files. The first file contains four perturbations, approximately a $5 \%, 10 \%, 20 \%$, and $30 \%$ change in the unperturbed tally. The remaining four input files contain the actual perturbations. In this way, it can be determined how well the perturbation feature predicts actual MCNP results (see Appendix A for a listing of the input titles).

\section{A. Criticality-Source Problems}

In this section, the KCODE feature is used to calculate $k_{e f f}$, and the macroscopic fission cross section is perturbed. The KCODE feature cannot calculate perturbed eigenvalues, so a track-length estimate for $\mathrm{k}_{\text {eff }}$ is used.

1. Godiva-Density Perturbation. In this problem, the density of the Godiva critical assembly, an unreflected sphere of highly enriched uranium, was increased from $18.74 \mathrm{~g} / \mathrm{cc}$ to 26 $\mathrm{g} / \mathrm{cc}$. A track-length estimate was used to calculate $k_{\text {eff. }}$ The unperturbed value for $k_{\text {eff }}$ was $0.99840 \pm 0.00080$, where the error given is a standard deviation. Table I gives the results from this problem, and the results are graphed in Fig. 1. As can be seen in Table I, the predicted values match the actual values, within statistics, up to about 5\%. After this point the second-order perturbation underestimates $\mathbf{k}_{\text {eff }}$. This bias is due to the inability of the differential operator, as implemented in MCNP, to calculate perturbed eigenfunctions. Essentially, the calculation of the perturbed eigenvalue is based on the eigenfunction at $18.74 \mathrm{~g} / \mathrm{cc}$.

2. Godiva-Composition Change. In this Problem, the composition of the Godiva critical assembly was perturbed from the original $94.73 \%$ U-235 5.27\% U-238 to 50\% of each isotope. The percents given are weight percents. A track-length estimate was used to calculate $k_{\text {eff }}$. The unperturbed value for $k_{\text {eff }}$ is again $0.99840 \pm 0.00080$. Table II gives the results from this problem, and Fig. 2 contains a graph of the results. The second-order perturbation accurately predicts, within statistics, the actual $k_{\text {eff }}$ estimator up to about $5 \%$, after which the second-order perturbation underestimates the actual $\mathrm{k}_{\mathrm{eff}}$ estimator. Again, this bias is due to the use of the unperturbed eigenfunction to calculate the perturbed eigenvalue. 
TABLE I

GODIVA DENSITY PERTURBATION $-k_{\text {eff }}$ ESTIMATE

\begin{tabular}{|l|c|c|c|c|}
\hline $\begin{array}{c}\text { Density } \\
(\mathrm{g} / \mathrm{cc})\end{array}$ & $\begin{array}{c}\text { Percent } \\
\text { Change } \\
\text { (Predicted) }\end{array}$ & $\begin{array}{c}\text { Standard } \\
\text { Deviation }\end{array}$ & $\begin{array}{c}\text { Percent } \\
\text { Change } \\
\text { (Actual) }\end{array}$ & $\begin{array}{c}\text { Standard } \\
\text { Deviation }\end{array}$ \\
\hline 20 & 5.283 & 0.016 & 5.41 & 0.12 \\
\hline 21 & 9.304 & 0.088 & 9.61 & 0.11 \\
\hline 23.5 & 18.696 & 0.059 & 19.29 & 0.12 \\
\hline 26 & 27.142 & 0.093 & 28.19 & 0.12 \\
\hline
\end{tabular}

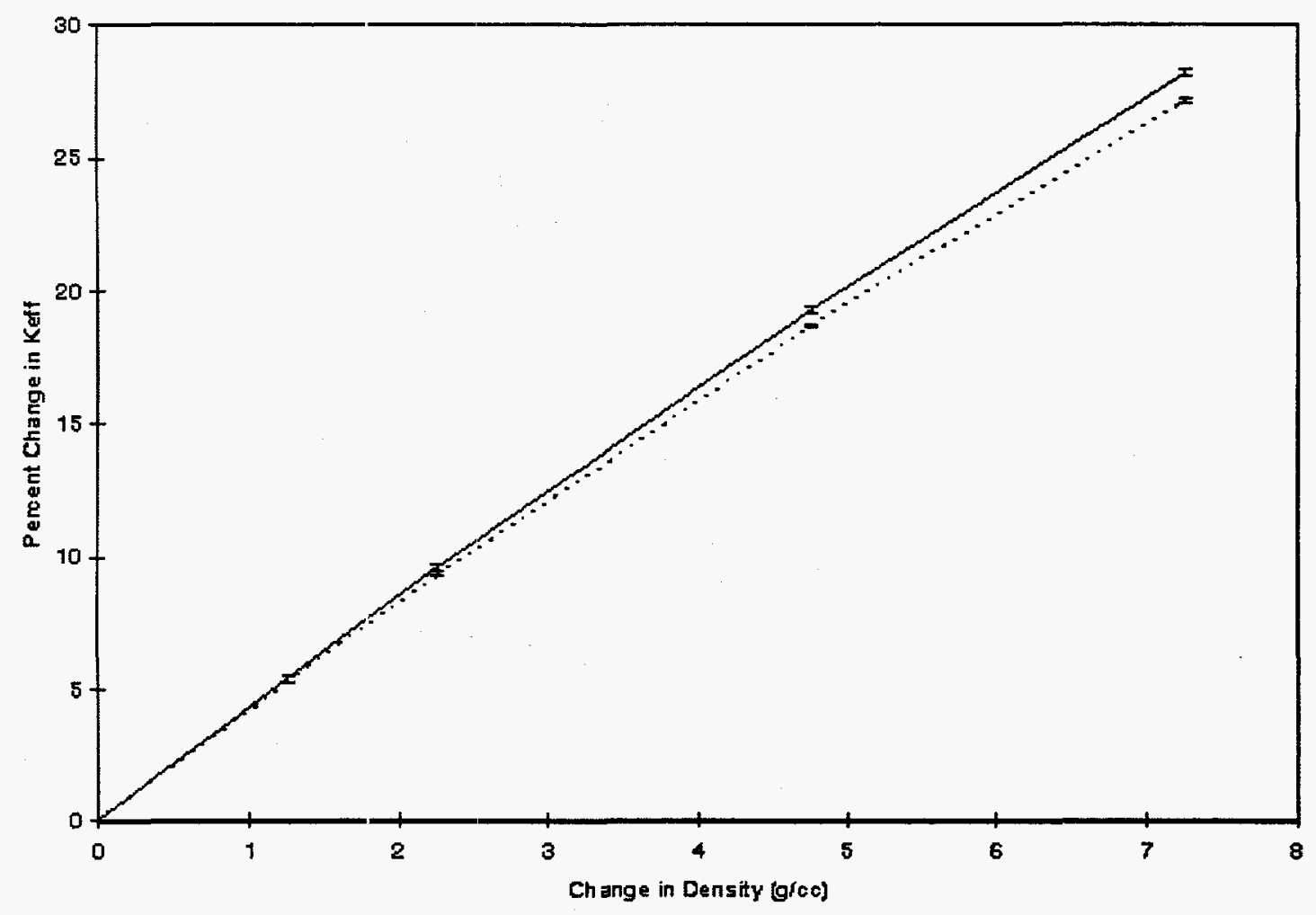

Fig. 1. Godiva-Density Perturbation. The predicted change is indicated by the dashed line; the actual change is indicated by the solid line. 
TABLE II

GODIVA COMPOSITION PERTURBATION $-k_{\text {eff }}$ ESTIMATE

\begin{tabular}{|c|c|c|c|c|}
\hline $\begin{array}{c}\text { U-238 } \\
\text { Weight } \\
\text { Fraction } \\
(\%)\end{array}$ & $\begin{array}{c}\text { Percent } \\
\text { Change } \\
\text { (Predicted) }\end{array}$ & $\begin{array}{c}\text { Standard } \\
\text { Deviation }\end{array}$ & $\begin{array}{c}\text { Percent } \\
\text { Change } \\
\text { (Actual) }\end{array}$ & $\begin{array}{c}\text { Standard } \\
\text { Deviation }\end{array}$ \\
\hline 13 & -4.307 & 0.082 & -4.31 & 0.11 \\
\hline 26 & -12.89 & 0.33 & -11.71 & 0.11 \\
\hline 38 & -22.31 & 0.74 & -19.54 & 0.10 \\
\hline 50 & -33.2 & 1.3 & -28.44 & 0.10 \\
\hline
\end{tabular}

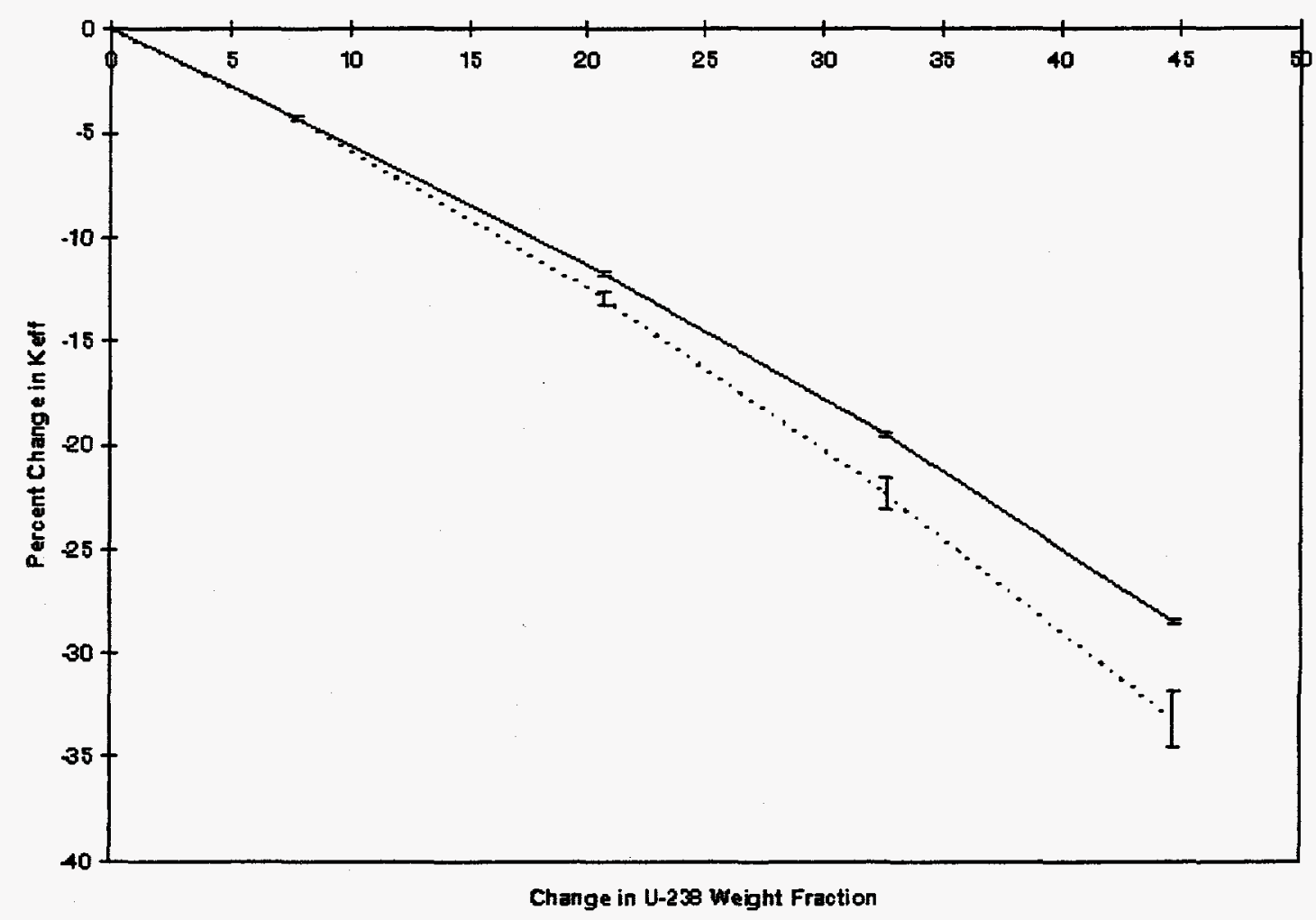

Fig. 2. Godiva-Composition Perturbation. The predicted change is indicated by the dashed line; the actual change is indicated by the solid line. 
3. Test Problem INP18- $\mathbb{K}_{\text {eff }}$ Estimate. This problem is a density perturbation of problem INP18 from the MCNP4B test suite. The input file is a hexagonal lattice core of a light water reactor. The density of the fuel was perturbed from the original $13.75 \mathrm{~g} / \mathrm{cc}$ to $26 \mathrm{~g} / \mathrm{cc}$. Again, a track-estimate of $k_{\text {eff }}$ was tallied. The unperturbed value for $k_{\text {eff }}$ is $1.0537 \pm 0.0011$. The results are presented in Table III and in Fig. 3. Again, the second-order perturbation is useful up to about $5 \%$. Notice in this problem that the discrepancy between the actual and predicted values increases at a greater rate as density is increased than in the Godiva examples. This difference is due to the fact that eigenfunctions are more easily perturbed by density and composition changes in reflected thermal systems than in bare fast systems. Thus, the difference between the actual and unperturbed eigenfunction in this case is expected to be greater than in a bare unreflected assembly, introducing more inaccuracy into the perturbation.

\section{B. Fixed-Source Problems}

In this section, fixed neutron, photon, and coupled neutron-photon problems are used to calculate various reaction rates with the cross section of interest being perturbed.

1. Test Problem INP02-Neutron Absorption Rate Estimate. This problem is a density perturbation of problem INP02 from the MCNP4B test suite. The input file is a large set of spheres with an inner region of boron surrounded by an aluminum shell. Within this aluminum shell is another set of spheres filled with aluminum. A neutron source is distributed in the boron sphere and has a uniform energy spectrum of 0.1 to $10 \mathrm{MeV}$. In this problem, the density of one of these spheres is perturbed from $2.7 \mathrm{~g} / \mathrm{cc}$ to $3.6 \mathrm{~g} / \mathrm{cc}$, and the neutron absorption rate is tallied. The unperturbed tally is $(6.58 \pm 0.11) \mathrm{E}-8$. The results from this problem are given in Table IV and in Fig. 4. As can be seen, the second-order perturbation predicts, within statistics, the actual MCNP tally up to at least a $30 \%$ increase in the unperturbed tally. Although the large error in the actual tally indicates that more histories should have been run, the corrected perturbation still predicts the tally well. 


\section{TABLE III}

TEST PROBLEM INP18 $-k_{\text {eff }}$ ESTIMATE

\begin{tabular}{|l|c|c|c|c|}
\hline $\begin{array}{c}\text { Density } \\
(\mathrm{g} / \mathrm{cc})\end{array}$ & $\begin{array}{c}\text { Percent } \\
\text { Change } \\
\text { (Predicted) }\end{array}$ & $\begin{array}{c}\text { Standard } \\
\text { Deviation }\end{array}$ & $\begin{array}{c}\text { Percent } \\
\text { Change } \\
\text { (Actual) }\end{array}$ & $\begin{array}{c}\text { Standard } \\
\text { Deviation }\end{array}$ \\
\hline 15.5 & 4.970 & 0.047 & 5.01 & 0.15 \\
\hline 17 & 8.735 & 0.084 & 9.09 & 0.15 \\
\hline 21.5 & 17.30 & 0.23 & 19.28 & 0.16 \\
\hline 26 & 21.78 & 0.50 & 27.50 & 0.16 \\
\hline
\end{tabular}

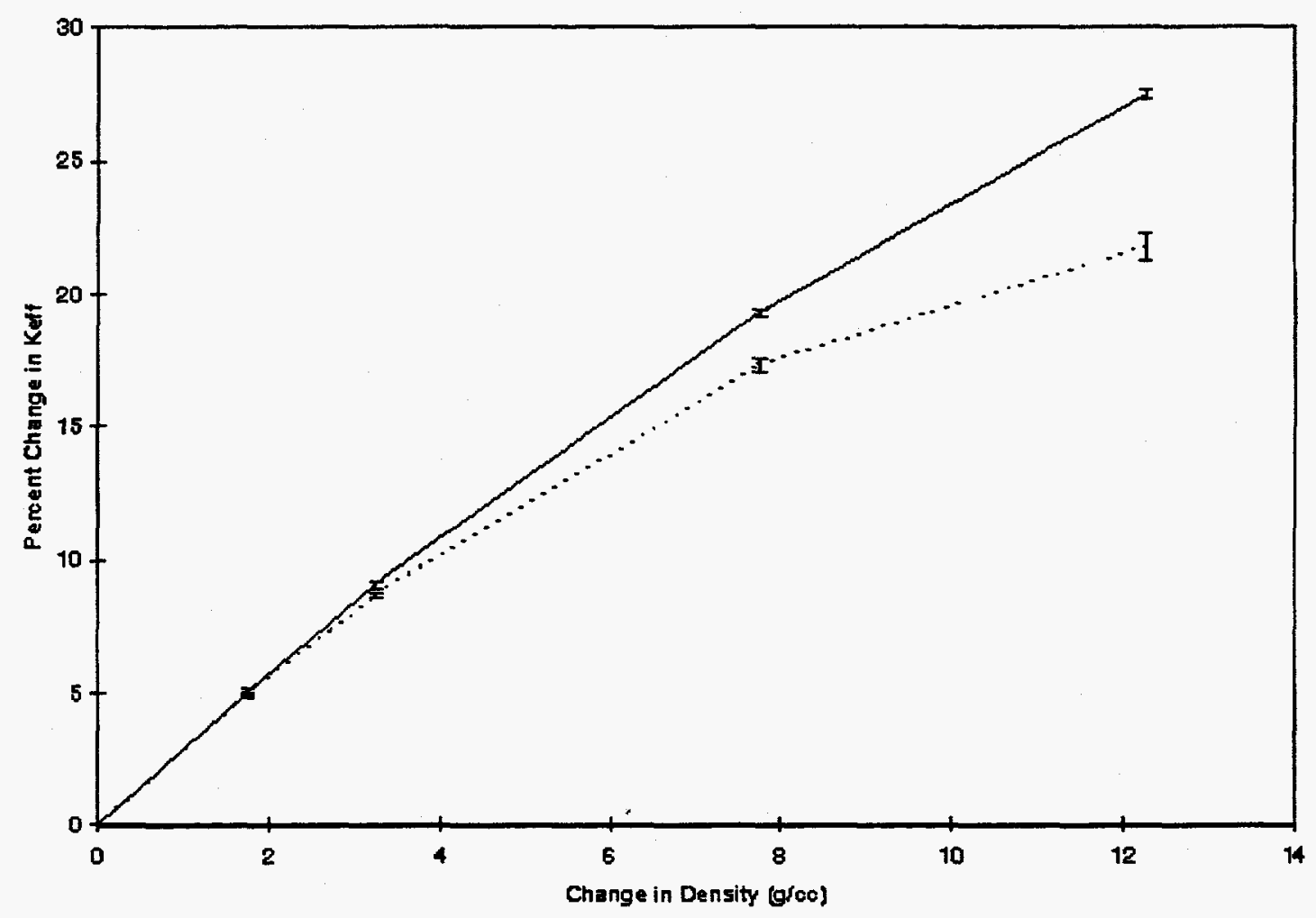

Fig. 3. Test Problem INP18- $k_{\text {eff }}$ Estimate. The predicted change is indicated by the dashed line; the actual change is indicated by the solid line. 


\section{TABLE IV}

TEST PROBLEM INP02 - NEUTRON ABSORPTION RATE ESTIMATE

\begin{tabular}{|c|c|c|c|c|}
\hline $\begin{array}{c}\text { Density } \\
(\mathrm{g} / \mathrm{cc})\end{array}$ & $\begin{array}{c}\text { Percent } \\
\text { Change } \\
\text { (Predicted) }\end{array}$ & $\begin{array}{c}\text { Standard } \\
\text { Deviation }\end{array}$ & $\begin{array}{c}\text { Percent } \\
\text { Change } \\
\text { (Actual) }\end{array}$ & $\begin{array}{c}\text { Standard } \\
\text { Deviation }\end{array}$ \\
\hline 2.85 & 5.61 & 0.14 & 5.6 & 2.5 \\
\hline 3 & 11.25 & 0.28 & 11.1 & 2.5 \\
\hline 3.3 & 22.52 & 0.56 & 22.0 & 2.7 \\
\hline 3.6 & 33.82 & 0.83 & 33.4 & 2.9 \\
\hline
\end{tabular}

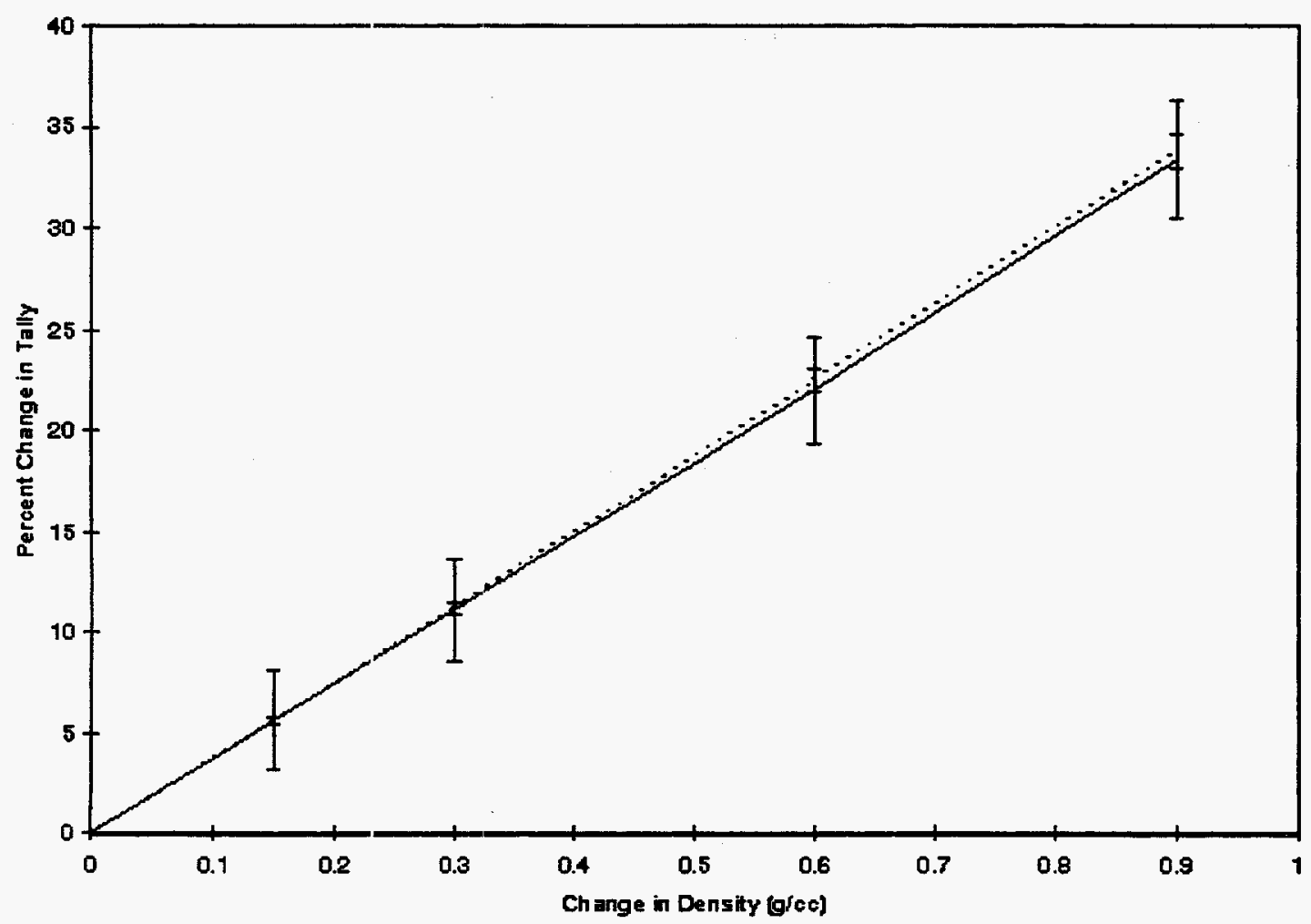

Fig. 4. Test Problem INP02-Neutron Absorption Rate Estimate. The predicted change is indicated by the dashed line; the actual change is indicated by the solid line. 
2. Test Problem INP04-Photon Collision Rate Estimate. This problem is a concentration perturbation of problem INP04 from the MCNP4B test suite. In this problem there is a large inner sphere composed of $\mathrm{UH}_{3}$ with an outer shell of $\mathrm{ULi}_{3}$. This sphere contains two smaller spheres of $\mathrm{ULi}_{3}$. A $3 \mathrm{MeV}$ photon point source is placed in the center of the large sphere, and the photon collision rate is calculated in the outer shell. The composition of the shell was perturbed from the original composition of $75 \%$ Lithium, $25 \%$ Uranium to $92.5 \%$ Lithium, $7.5 \%$ Uranium. The percents given are atom percents. The unperturbed tally for this problem is $(6.987 \pm 0.006) E-4$. The results from this problem are given in Table $V$ and in Fig. 5. As can be seen in Fig. 5, the corrected perturbation does not predict the actual values very well. Let us examine the perturbation more closely. Values for the uncorrected first and total perturbation of the lithium bin are given in Table VI. Notice for the $5 \%$ perturbation $(77.5 \% \mathrm{Li})$ that the total perturbation is approximately ten percent larger than the first-order perturbation. This magnitude represents the limit of the differential operator technique as implemented in MCNP. Having a second-order term that is ten percent of the first-order term indicates that some higher order terms are needed to predict the tally accurately. The situation is worse with the $30 \%$ increase in the unperturbed tally $(92.5 \% \mathrm{Li})$. For this perturbation the second-order term is almost equal to that of the first-order term. This problem demonstrates that the perturbation feature cannot accurately predict every tally, and a second-order Taylor series approximation will fail if the tally exhibits higher than second-order behavior.

3. Test Problem INP10-Photon Collision Rate Estimate. This problem is a density perturbation of problem INP10 from the MCNP4B test suit. The geometry of this problem consists of two concentric infinite cylinders. The inner cylinder is filled with water, and the outer shell is filled with copper. Near the origin the inner cylindrical region is separated into several disks filled with water, carbon, and one with void. The disk with the void contains a cube of $\mathrm{CuO}$, and an adjacent disk of water contains a void torus surrounded by a shell of copper. A Watt fission spectrum neutron source is distributed evenly in the $\mathrm{CuO}$ cube, and the photon collision rate is tallied in one of the carbon disks. The density of this disk was perturbed from the original $2.25 \mathrm{~g} / \mathrm{cc}$ to $3.6 \mathrm{~g} / \mathrm{cc}$, and the unperturbed tally is $(6.64 \pm 0.02) \mathrm{E}-6$. The results from this problem are given in Table VII and in Fig. 6. 


\section{TABLE V}

TEST PROBLEM INP04 — PHOTON COLLISION RATE ESTIMATE

\begin{tabular}{|c|c|c|c|c|}
\hline $\begin{array}{c}\text { Li Atom } \\
\text { Fraction } \\
(\%)\end{array}$ & $\begin{array}{c}\text { Percent } \\
\text { Change } \\
\text { (Predicted) }\end{array}$ & $\begin{array}{c}\text { Standard } \\
\text { Deviation }\end{array}$ & $\begin{array}{c}\text { Percent } \\
\text { Change } \\
\text { (Actual) }\end{array}$ & $\begin{array}{c}\text { Standard } \\
\text { Deviation }\end{array}$ \\
\hline 77.5 & -0.726 & 0.022 & -1.97 & 0.12 \\
\hline 82.5 & -2.921 & 0.083 & -7.85 & 0.12 \\
\hline 87.5 & -6.11 & 0.17 & -18.35 & 0.11 \\
\hline 92.5 & -10.28 & 0.34 & -37.60 & 0.11 \\
\hline
\end{tabular}

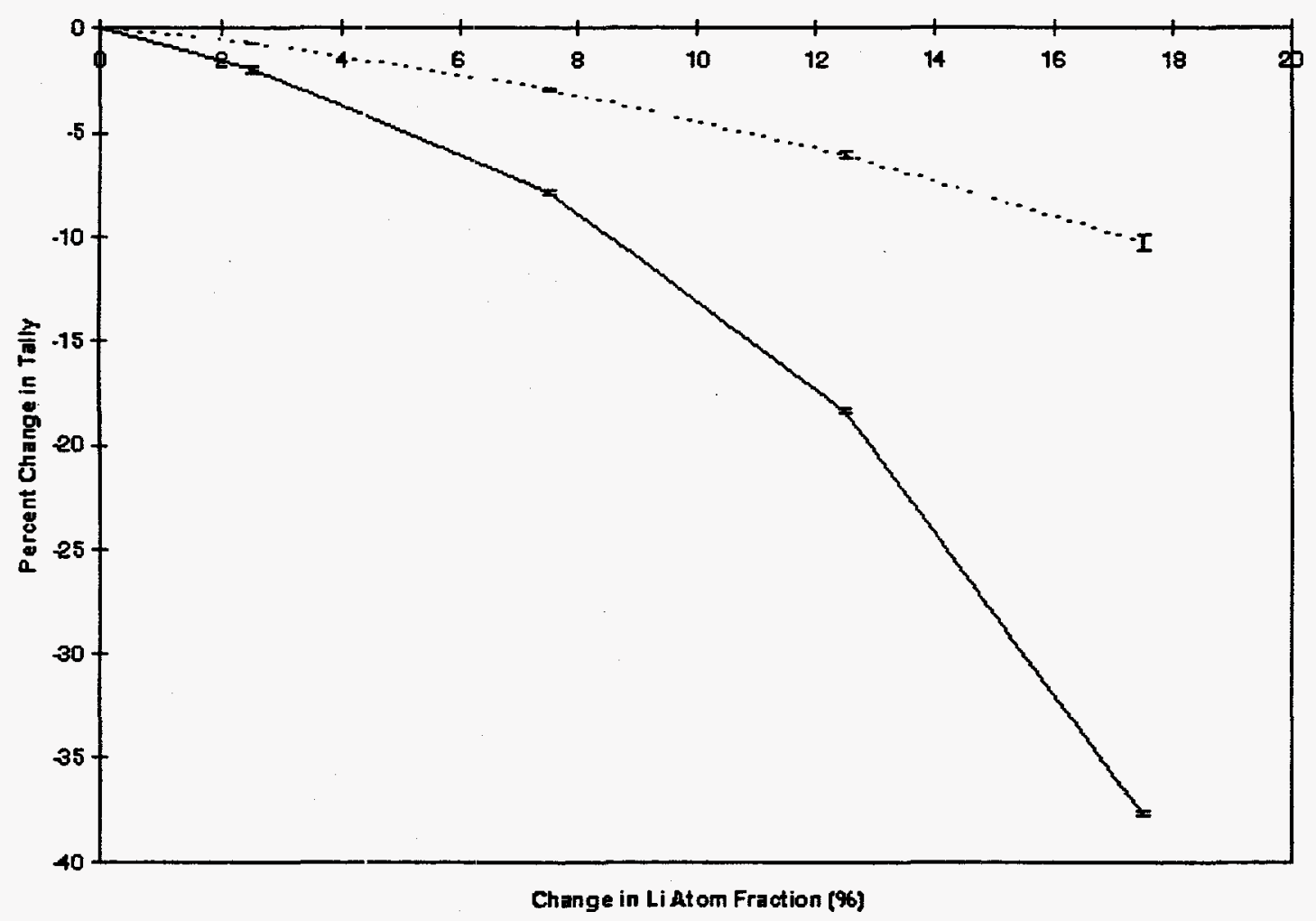

Fig. 5. Test Problem INP04-Photon Collision Rate Estimate. The predicted change is indicated by the dashed line; the actual change is indicated by the solid line. 


\section{TABLE VI}

\section{LITHIUM BIN UNCORRECTED PERTURBATIONS}

\begin{tabular}{|c|c|c|}
\hline $\begin{array}{c}\text { Li Atom } \\
\text { Fraction } \\
(\%)\end{array}$ & $\begin{array}{c}\text { First-Order } \\
\text { Uncorrected } \\
\text { Perturbation }\end{array}$ & $\begin{array}{c}\text { Total } \\
\text { Uncorrected } \\
\text { Perturbation }\end{array}$ \\
\hline 77.5 & $6.68556 \mathrm{E}-7$ & $7.21353 \mathrm{E}-7$ \\
\hline 92.5 & $4.67989 \mathrm{E}-6$ & $7.26697 \mathrm{E}-6$ \\
\hline
\end{tabular}

Again, the second-order corrected perturbation predicts the actual MCNP values well up to a $30 \%$ increase in the unperturbed tally. Although the perturbation begins to diverge from the actual value after about a $10 \%$ increase in the unperturbed tally, the predicted values agree with the actual values, within statistics, up to $30 \%$.

\section{RECOMMENDATIONS}

The corrections presented here allow the users of MCNP4B to accurately calculate perturbations in cross-section dependent tallies. It is recommended that the perturbation routines in MCNP be rewritten to fully account for any cross-section dependent tallies. In addition, it is recommended that a method of perturbing eigenfunctions be developed for criticality problems. This capability will allow for the calculation of perturbed eigenvalues with the same confidence as fixed source problems.

\section{SUMMARY}

The recently released MCNP4B employs the differential operator technique to calculate perturbations in tallies caused by changes in cross-section data. The implementation of this technique may cause incorrect results to be generated if the tally is dependent on perturbed crosssection data. To extend its generality, additional terms must be added to the perturbation that account for this dependence. Corrections to both the first- and second-order perturbations are derived and demonstrated for various types of perturbations. In addition, the correction is verified with six test problems covering criticality and fixed sources. 
TABLE VII

TEST PROBLEM INP10 - PHOTON COLLISION RATE ESTIMATE

\begin{tabular}{|c|c|c|c|c|}
\hline $\begin{array}{c}\text { Density } \\
(\mathrm{g} / \mathrm{cc})\end{array}$ & $\begin{array}{c}\text { Percent } \\
\text { Change } \\
\text { (Predicted) }\end{array}$ & $\begin{array}{c}\text { Standard } \\
\text { Deviation }\end{array}$ & $\begin{array}{c}\text { Percent } \\
\text { Change } \\
\text { (Actual) }\end{array}$ & $\begin{array}{c}\text { Standard } \\
\text { Deviation }\end{array}$ \\
\hline 2.4 & 4.80 & 0.14 & 4.49 & 0.50 \\
\hline 2.6 & 11.01 & 0.48 & 10.69 & 0.52 \\
\hline 3 & 22.8 & 1.9 & 20.83 & 0.54 \\
\hline 3.6 & 38.8 & 5.9 & 32.83 & 0.57 \\
\hline
\end{tabular}

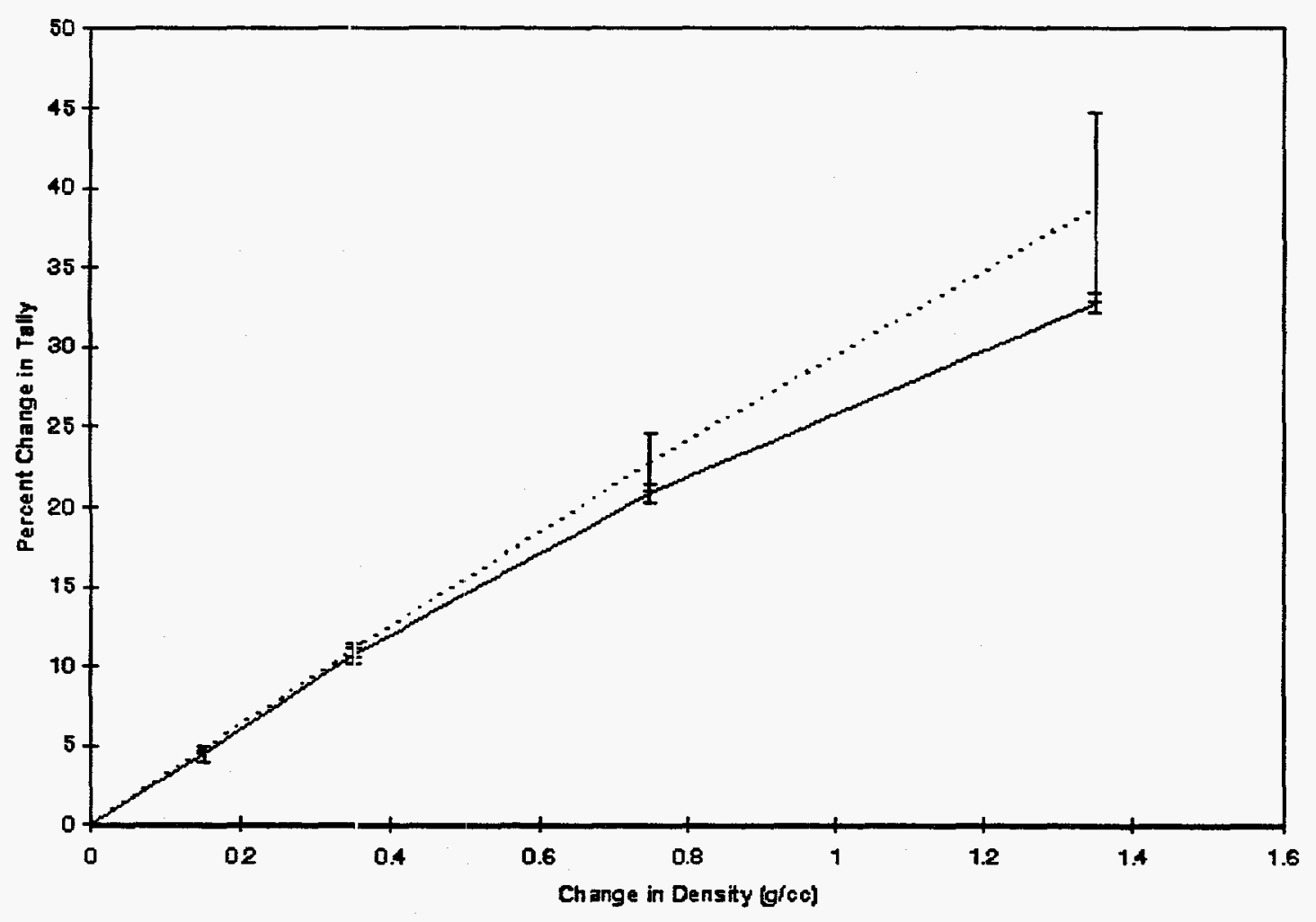

Fig. 6. Test Problem INP10-Photon Collision Rate Estimate. The predicted change is indicated by the dashed line; the actual change is indicated by the solid line. 


\section{REFERENCES}

1. J. Briesmeister, Editor, "MCNPTM - A General Monte Carlo N-Particle Transport Code," LA-12625-M (March 1997).

2. G. W. McKinney, "Theory Related to the Differential Operator Perturbation Technique," Los Alamos National Laboratory memo, X-6:GWM-94-124 (1994).

3. G. W. McKinney and J. L. Iverson, "Verification of the Monte Carlo Differential Operator Technique for MCNP," Los Alamos National Laboratory report, LA-13098 (February 1996). 


\section{APPENDIX A}

\section{INPUT FILES}

The input files for the test problems in Section IV, "Verification Results," are listed. For each example five input files are needed. The first file contains four perturbations and gives the unperturbed result. The remaining four files give the "true" MCNP results for the perturbations by running with the perturbed densities and/or concentrations. To save space, only the first input file for each example is listed. The remaining four input files are the same except for the changes noted.

\section{GODIVA-DENSITY PERTURBATION}

The input for the unperturbed problem, input=godiva1, is

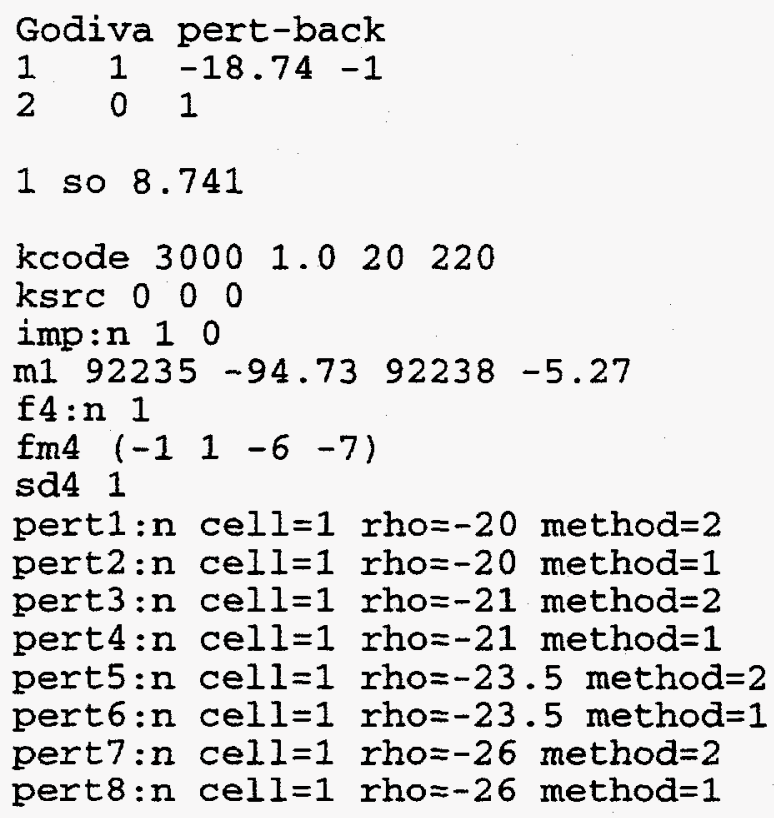

The "true" results for the four perturbations are obtained by deleting the PERT cards and changing the density of cell 1 on line 2 ,

$$
\begin{array}{lllll}
1 & 1 & -18.74 & -1
\end{array}
$$

with the first perturbation, input=godiva2,

$$
\begin{array}{llll}
1 & 1 & -20 & -1
\end{array}
$$


or with the second perturbation, input=godiva3,

$\begin{array}{llll}1 & 1 & -21 & -1\end{array}$

or with the third perturbation, input=godiva4,

$\begin{array}{lllll}1 & 1 & -23.5 & -1\end{array}$

or with the fourth perturbation, input=godiva5,

$\begin{array}{llll}1 & 1 & -26 & -1\end{array}$

II. GODIVA-COMPOSITION CHANGE

The input for the unperturbed problem, input=gconc1, is

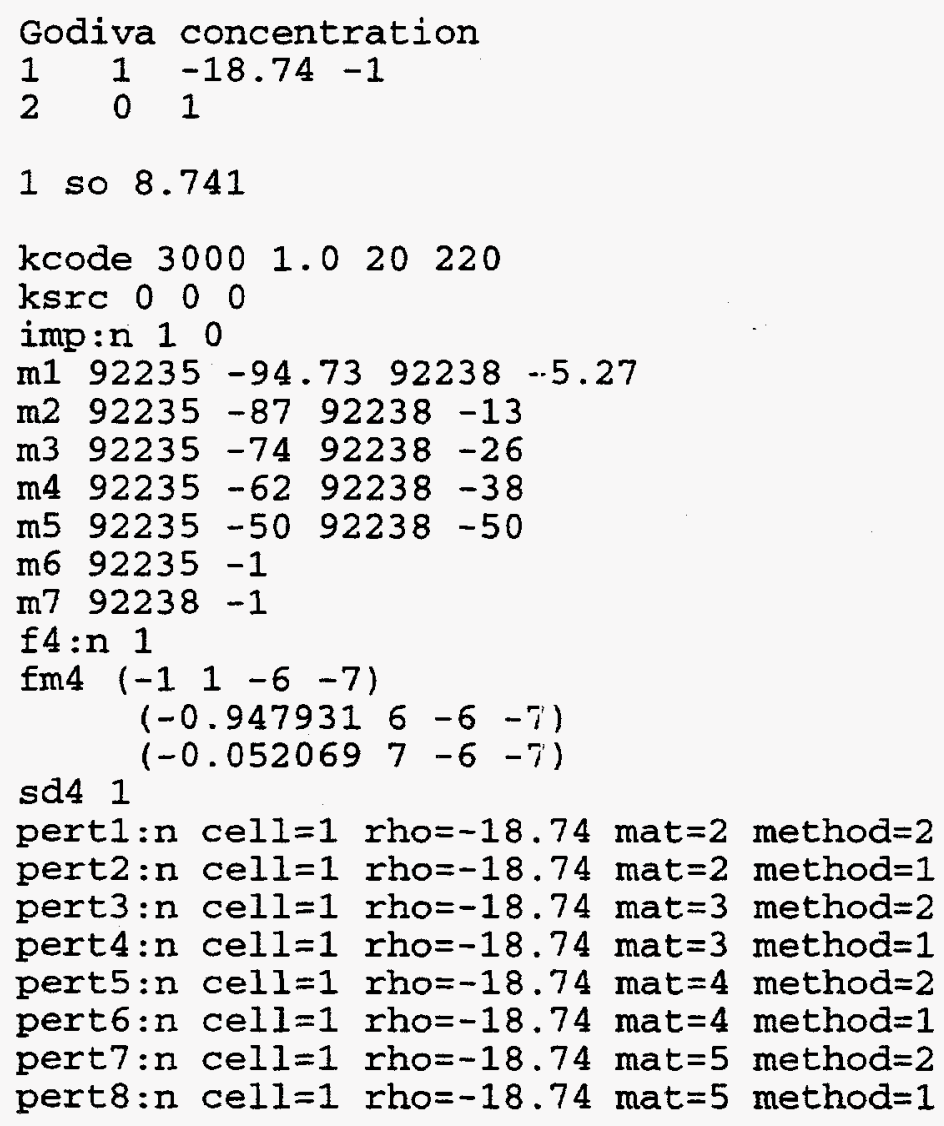

The "true" results for the four perturbations are obtained by deleting the PERT cards and replacing material 1 of cell 1 (line 2) and the FM card (lines $18-20$ ),

$\begin{array}{lllll}1 & 1 & -18.74 & -1\end{array}$ 


$$
\begin{aligned}
& \text { fm4 (-1 } 1 \text { - } 1-6 \quad-7) \\
& \left(\begin{array}{lllll}
-0.947931 & 6 & -6 & -7
\end{array}\right) \\
& \left(\begin{array}{lllll}
-0.052069 & 7 & -6 & -7
\end{array}\right)
\end{aligned}
$$

with the first perturbation using material 2 , input=gconc2,

$$
\begin{array}{lllll}
1 & 2 & -18 & 74 & -1 \\
\text { fm4 } & \left(\begin{array}{llll}
-1 & 2 & -6 & -7
\end{array}\right)
\end{array}
$$

or with the second perturbation using material 3, input=gconc3,

$$
\begin{array}{lllll}
1 & 3 & -18 & 74 & -1 \\
\text { fm4 } & \left(\begin{array}{llll}
-1 & 3 & -6 & -7
\end{array}\right)
\end{array}
$$

or with the third perturbation using material 4 , input=gconc4,

$$
\begin{array}{lllll}
1 & 4 & -18 & 74 & -1 \\
\text { fm4 } & \left(\begin{array}{llll}
-1 & 4 & -6 & -7
\end{array}\right)
\end{array}
$$

or with the fourth perturbation using material 5 , input=gconc5,

$$
\begin{array}{llll}
1 & 5 & -18.74 & -1 \\
\text { fm4 } & \left(\begin{array}{llll}
-1 & 5 & -6 & -7
\end{array}\right)
\end{array}
$$

\section{TEST PROBLEM INP18-K $\mathrm{K}_{\text {eff }}$ ESTIMATE}

The input for the unperturbed problem, input=inp 181 , is

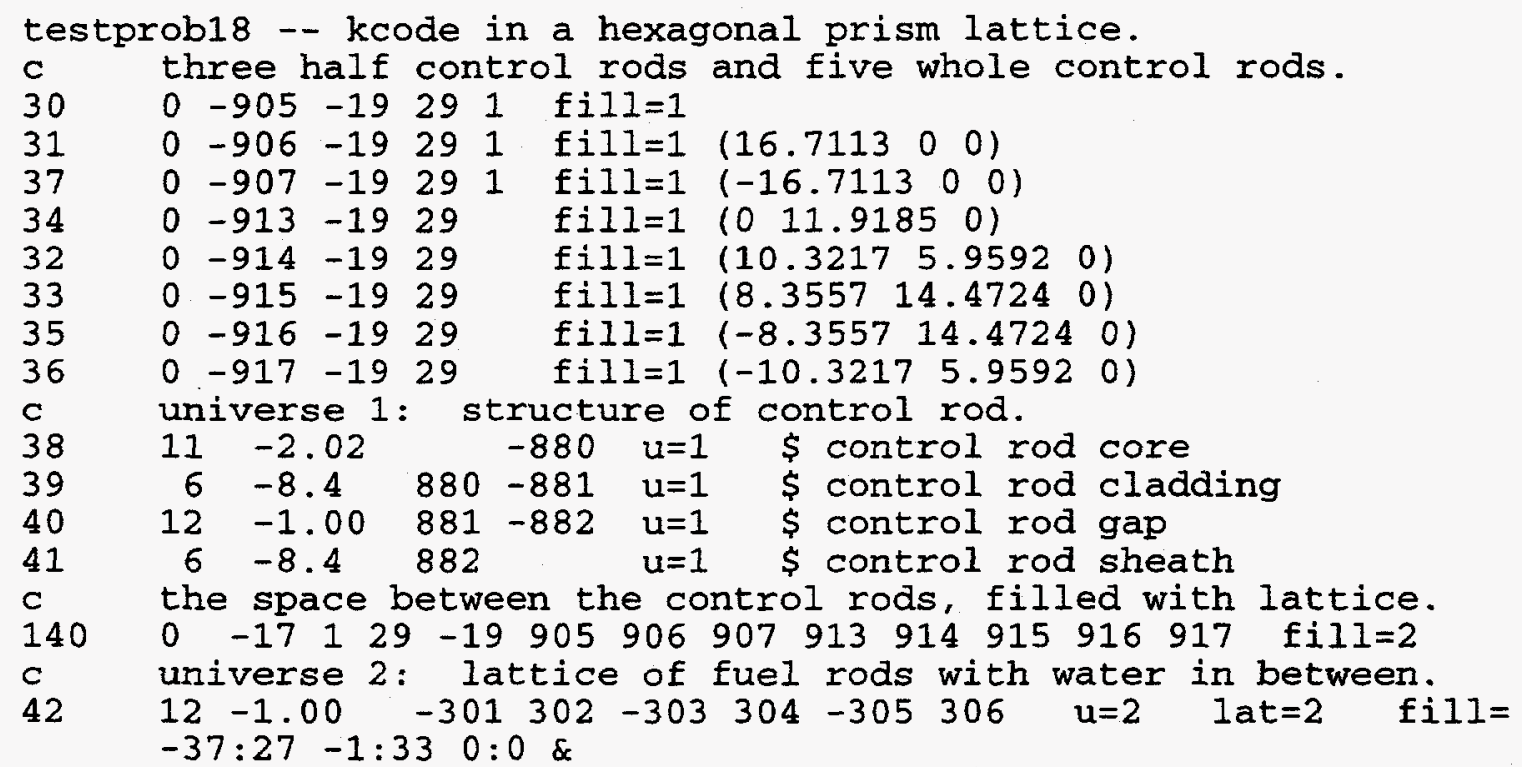




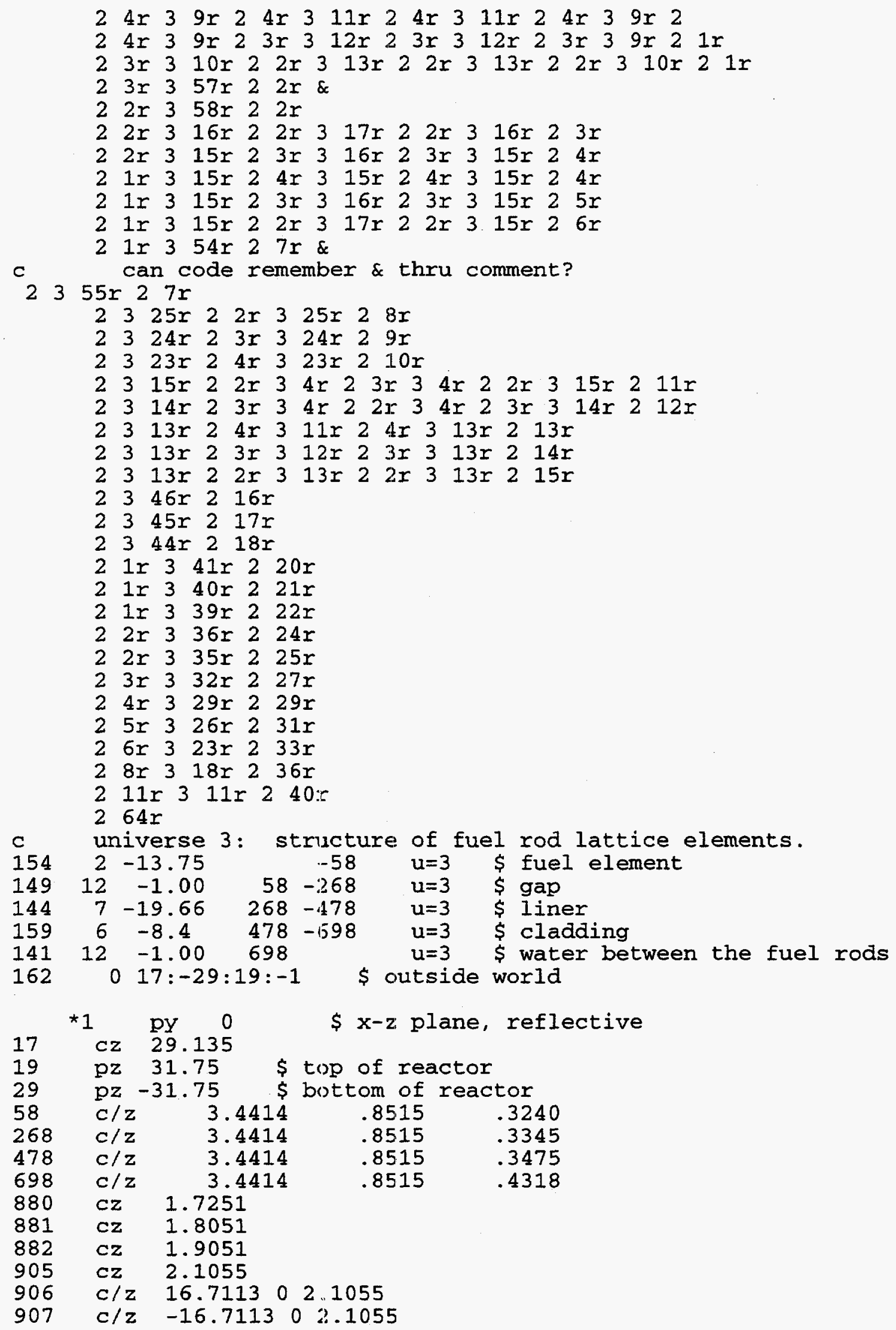




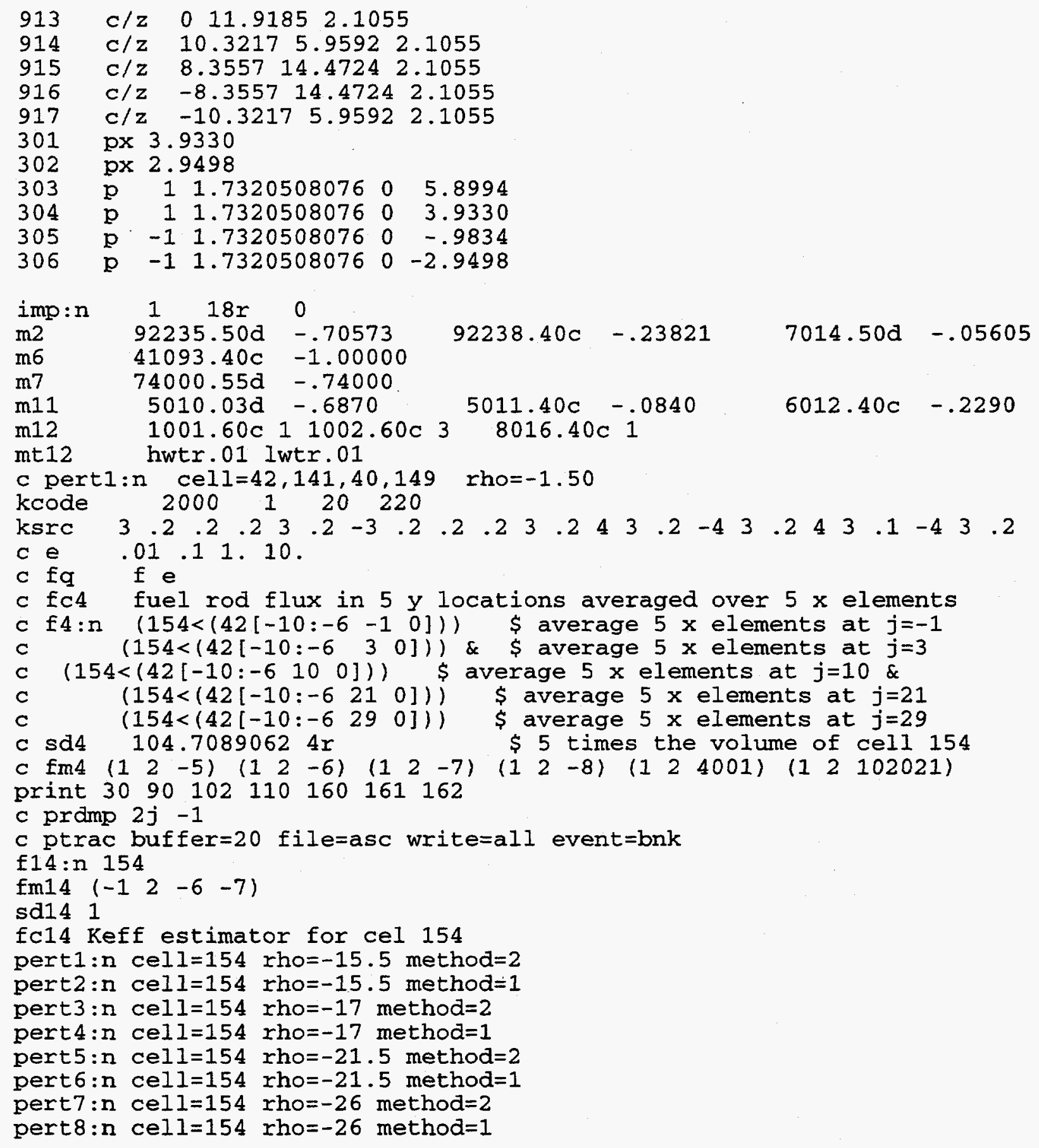

The "true" results for the four perturbations are obtained by deleting the PERT cards and replacing the density of material 2 , cell 154 , line 58 ,

$$
1542-13.75 \quad-58 \quad u=3 \quad \text { fuel element }
$$

with the first perturbation, input=inp182,

$1542-15.5 \quad-58 \quad u=3 \quad$ \& fuel element 
or with the second perturbation, input=inp183,

$1542-17 . \quad-58 \quad u=3 \quad$ fuel element

or with the third perturbation, input=inp184,

$1542-21.5 \quad-58 \quad u=3 \quad \$$ fuel element

or with the fourth perturbation, input=inp185,

$1542-26 . \quad-58 \quad u=3 \quad \$$ fuel element

IV. TEST PROBLEM INP02-NEUTRON ABSORPTION RATE ESTIMATE

The input for the unpelturbed problem, input=inp021, is

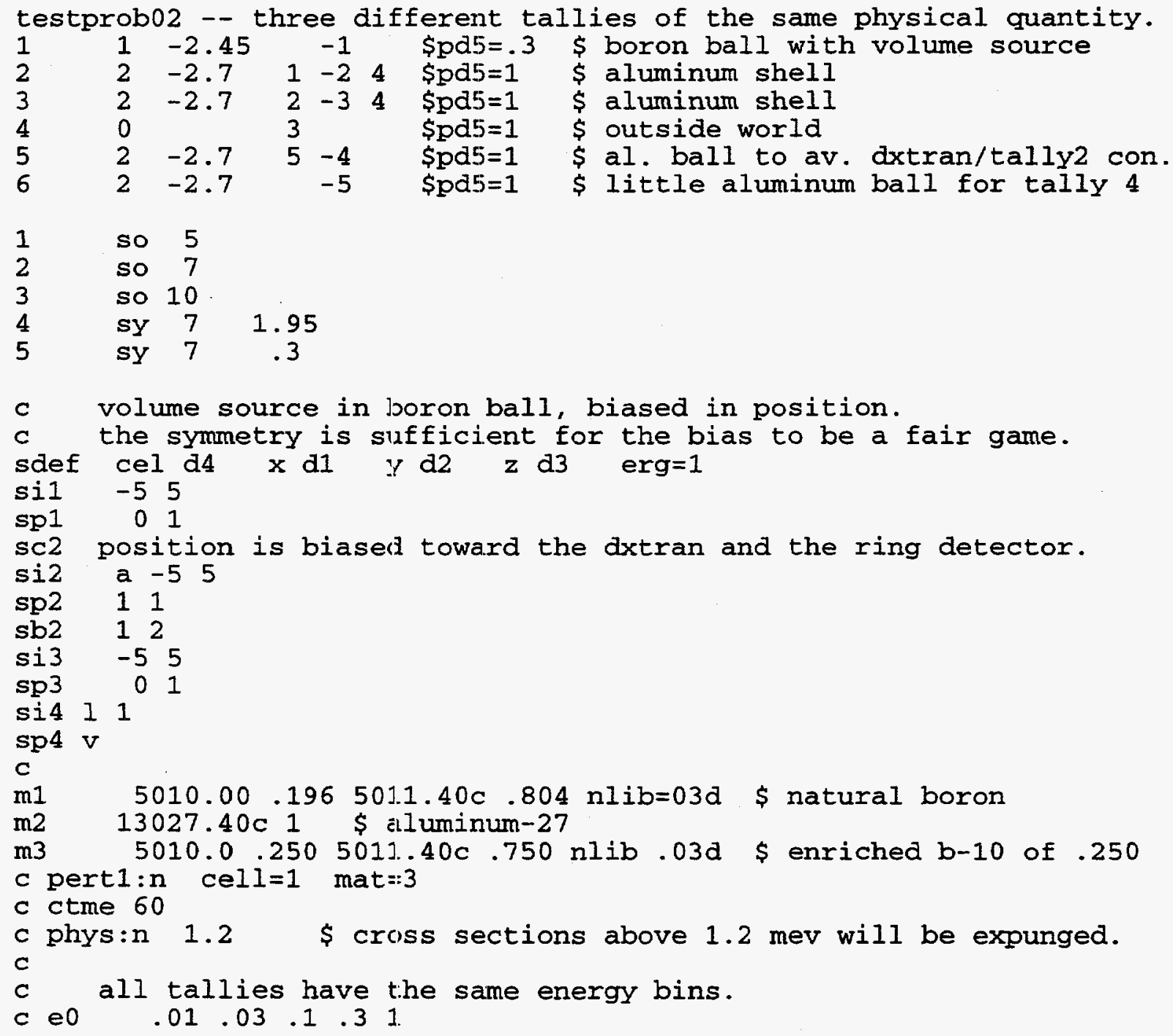


c fc2 average flux on surface 2 .

c $f 2: n \quad 2$

C $\mathrm{f} 1: \mathrm{n} 1$

c $c 1 \quad-.8 \quad 3 i \quad 0 \quad 4 i 1 t$

c fql $c$ e

c tfl $17 x$

c ft2 tmc -2.05

c $t 2 \quad-2 \quad 9 i \quad 3 \quad 10 \quad 100$

$c \mathrm{fq} 2 \mathrm{t} e$

c ft1 frv 345 geb 120

fc4 neutron absorption in cell 6

$\mathrm{f} 4: \mathrm{n} \quad 6$

fm4 $\left(\begin{array}{lll}-1 & 2 & -2\end{array}\right)$

c dd5 .003

c fq5 u e

c fu5 $18 i 10$

c ft5 inc

c fc5 average flux at ring detector.

c fy5:n $5 \quad 4.89 .7$

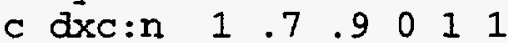

c dd1 .04 100

thtme $\begin{array}{rrrrr}-10 & 0 & .5 & 1 & 2\end{array}$

\# tmp1 tmp2 tmp3 tmp4 tmp5

$1 \quad 1 e-8 \quad 2 e-8 \quad 3 e-8 \quad 4 e-8 \quad 5 e-8$

$2 \quad 2 e-8 \quad 3 e-8 \quad 5 e-8 \quad 4 e-8 \quad 3 e-8$

$3 \quad 1 e-8 \quad 5 e-8 \quad 4 e-8 \quad 3 e-8 \quad 2 e-8$

$\begin{array}{llllll}4 & 0 & 0 & 0 & 0 & 0\end{array}$

$5 \quad 2 e-8 \quad 1 e-8 \quad 5 e-8 \quad 3 e-8 \quad 1 e-8$

c

$6 \quad 3 e-8 \quad 2 e-8 \quad 1 e-8 \quad 2 e-8 \quad 1 e-8$

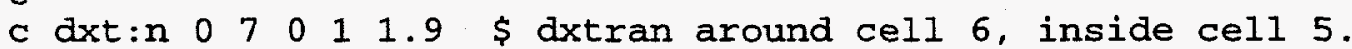
imp:n 111110001

c

nps 10000000

print $1072 \quad 170$

c prdmp $2500625-12 \quad$ \$ print metal file.

c dbcn $14 j 1$

c ptrac buffer $=2$ file=asc event $=\operatorname{src} n p s=1,200$ cell $1=3$

pert $1: \mathrm{n}$ cell=6 rho=-2.85 method=2

pert $2: n$ cell $1=6$ rho $=-2.85$ method $=1$

pert $3: \mathrm{n}$ cell=6 rho $=-3$ method $=2$

pert $4: n$ cell $1=6$ rho $=-3$ method $=1$

pert $5: \mathrm{n}$ cell $1=6$ rho $=-3.3$ method $=2$

pert $6: n$ cell $=6$ rho $=-3.3$ method $=1$

pert $7: n$ cell $=6$ rho $=-3.6$ method $=2$

pert $8: n$ cell=6 $r h o=-3.6$ method $=1$

The "true" results for the four perturbations are obtained by deleting the PERT cards and changing the density of cell 6 , material 2 , line 7 ,

$$
62-2.7 \quad-5 \quad \$ p d 5=1 \quad \$ \text { little aluminum ball for tally } 4
$$

with the first perturbation, input=inp022,

$$
62-2.85 \quad-5 \quad \$ p d 5=1 \quad \$ \text { little aluminum ball for tally } 4
$$


or with the second perturbation, input=inp023,

$6 \quad 2-3 . \quad-5 \quad \$ p a 5=1 \quad \$$ little aluminum ball for tally 4

or with the third perturbation, input=inp024,

$6 \quad 2-3.3 \quad-5 \quad$ \$pd5=1 \$ little aluminum ball for tally 4

or with the fourth perturbation, input=inp 025 ,

$6 \quad 2-3.6 \quad-5 \quad \$ p d 5=1 \quad$ \$ little aluminum ball for tally 4

V. TEST PROBLEM INP04-PHOTON COLLISION RATE ESTIMATE

The input for the unperturbed problem, input=inp041, is

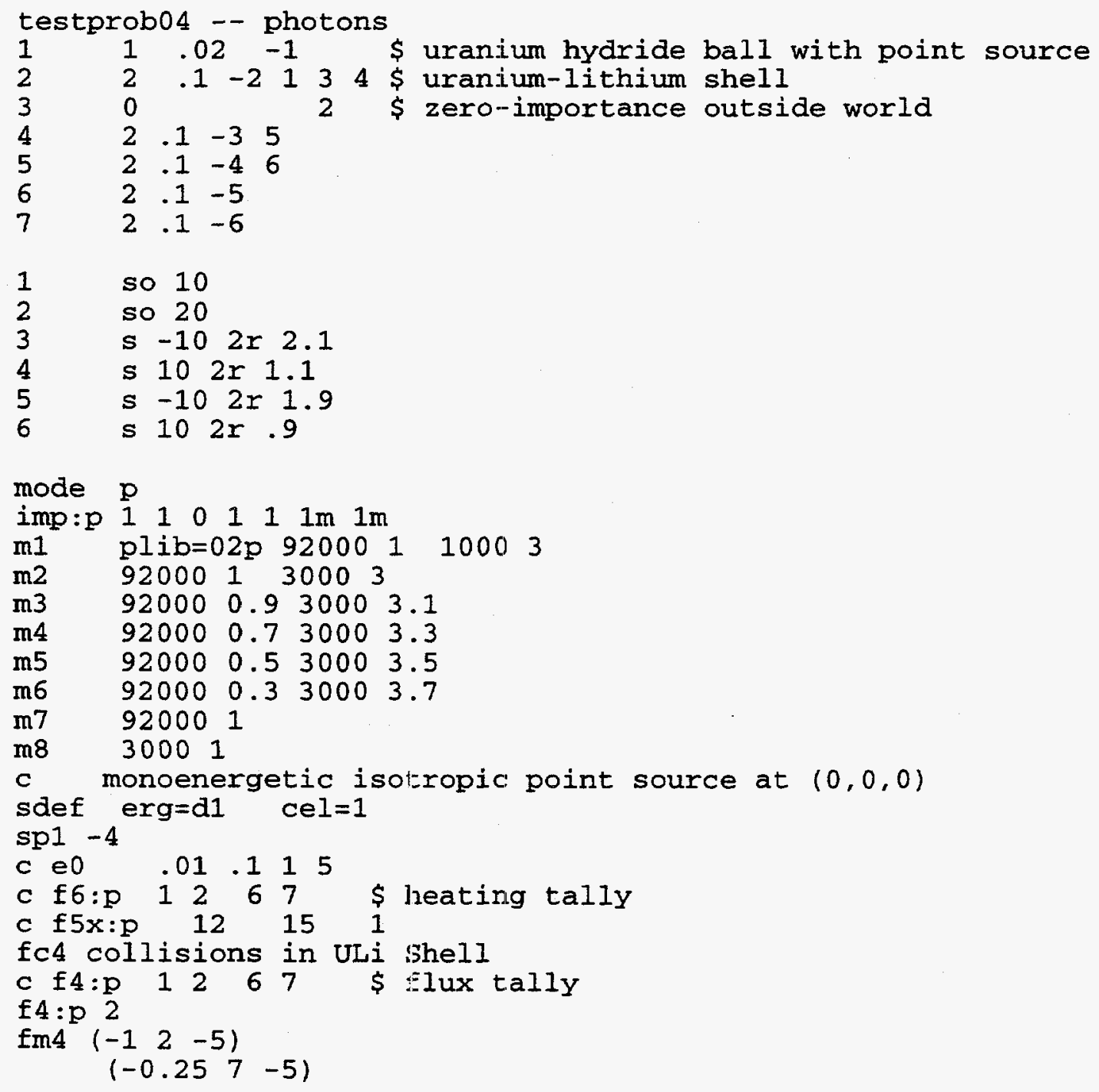




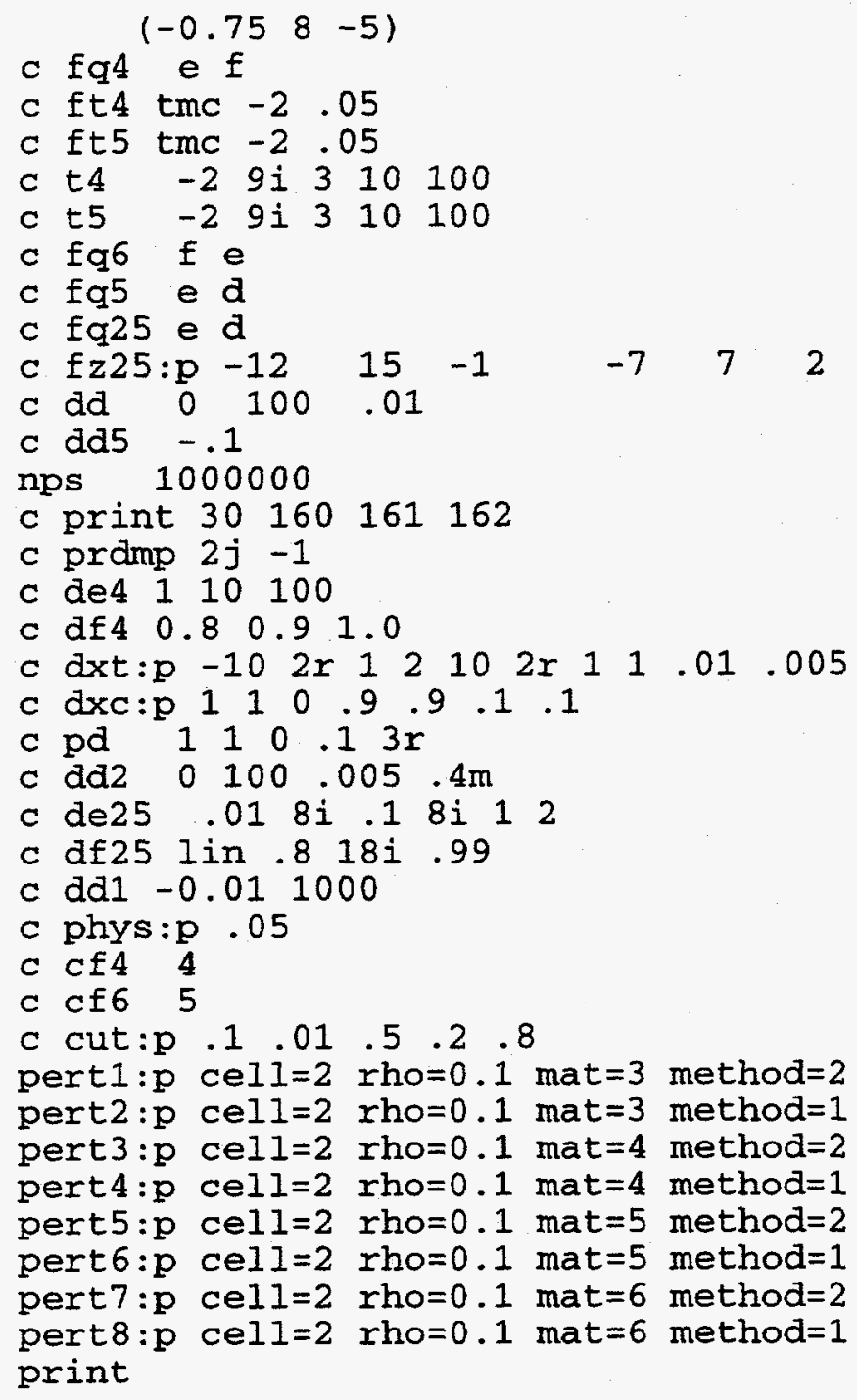

The "true" results for the four perturbations are obtained by deleting the PERT cards and replacing material 2 of cell 2 (line 3) and the FM card (lines 36 - 38),

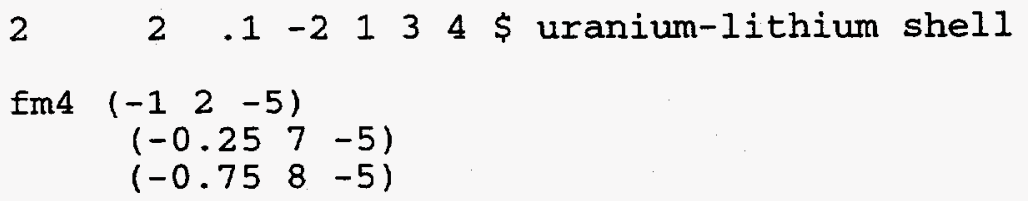

with the first perturbation using material 3, input=inp042,

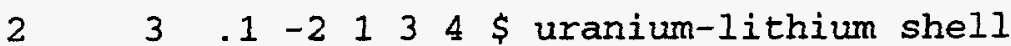

$$
\begin{aligned}
& \text { fm4 }\left(\begin{array}{lll}
-1 & 3 & -5
\end{array}\right)
\end{aligned}
$$

or with the second perturbation using material 4 , input=inp043, 
fm4 $\left(\begin{array}{lll}-1 & 4 & -5\end{array}\right)$

or with the third perturbation using material 5, input=inp044,

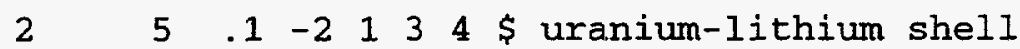

fm4 $\left(\begin{array}{lll}-1 & 5 & -5\end{array}\right)$

or with the fourth perturbation using material 6, input=inp045,

$\begin{array}{llllllll}2 & 6 & . & -2 & 1 & 3 & 4 & \$\end{array}$ uranium-lithium shell

fm4 $\left(\begin{array}{lll}-1 & 6 & -5\end{array}\right)$

VI. TEST PROBLEM INP10-PHOTON COLLISION RATE ESTIMATE

The input for the unperturbed problem, input=inp101, is

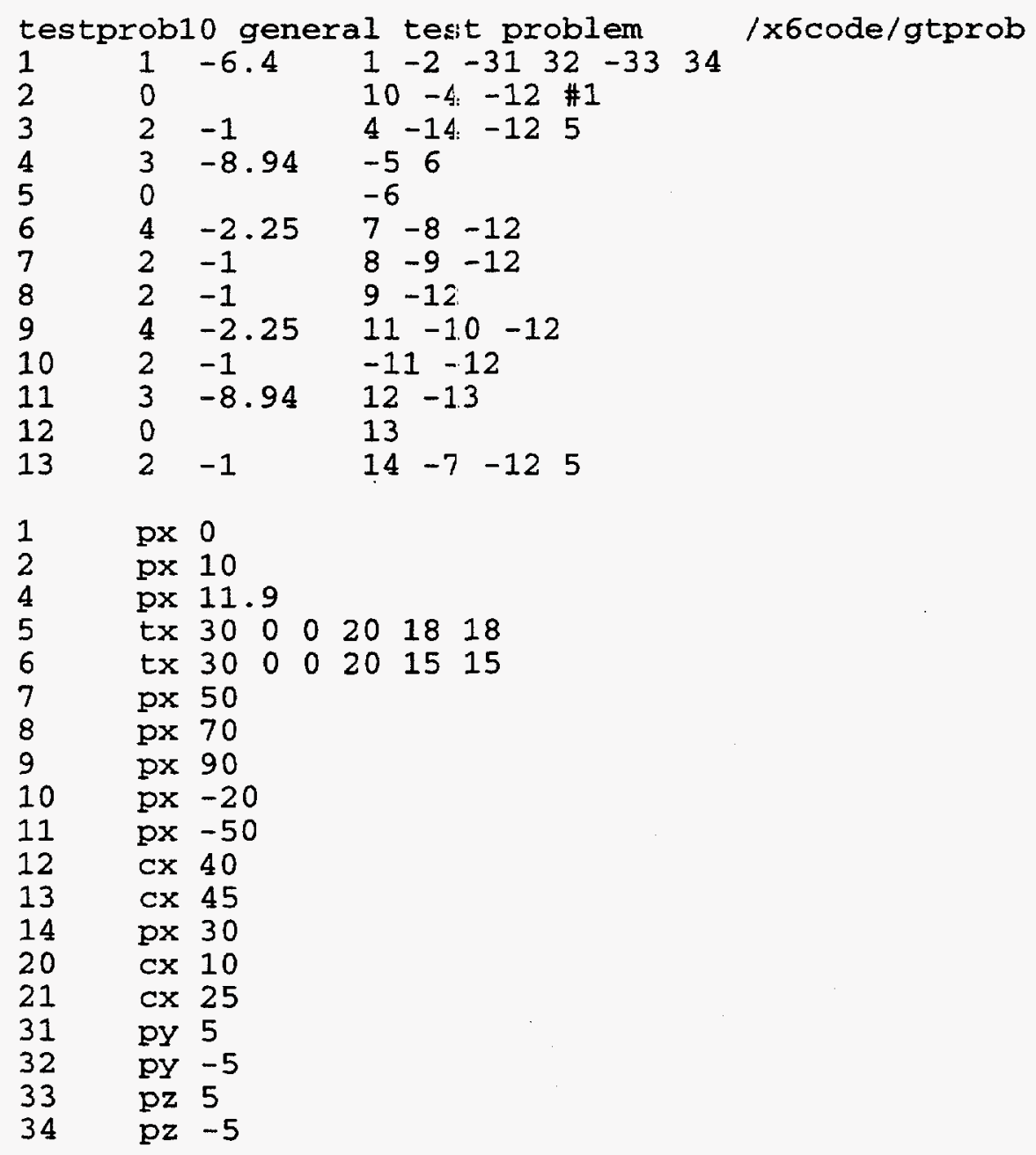




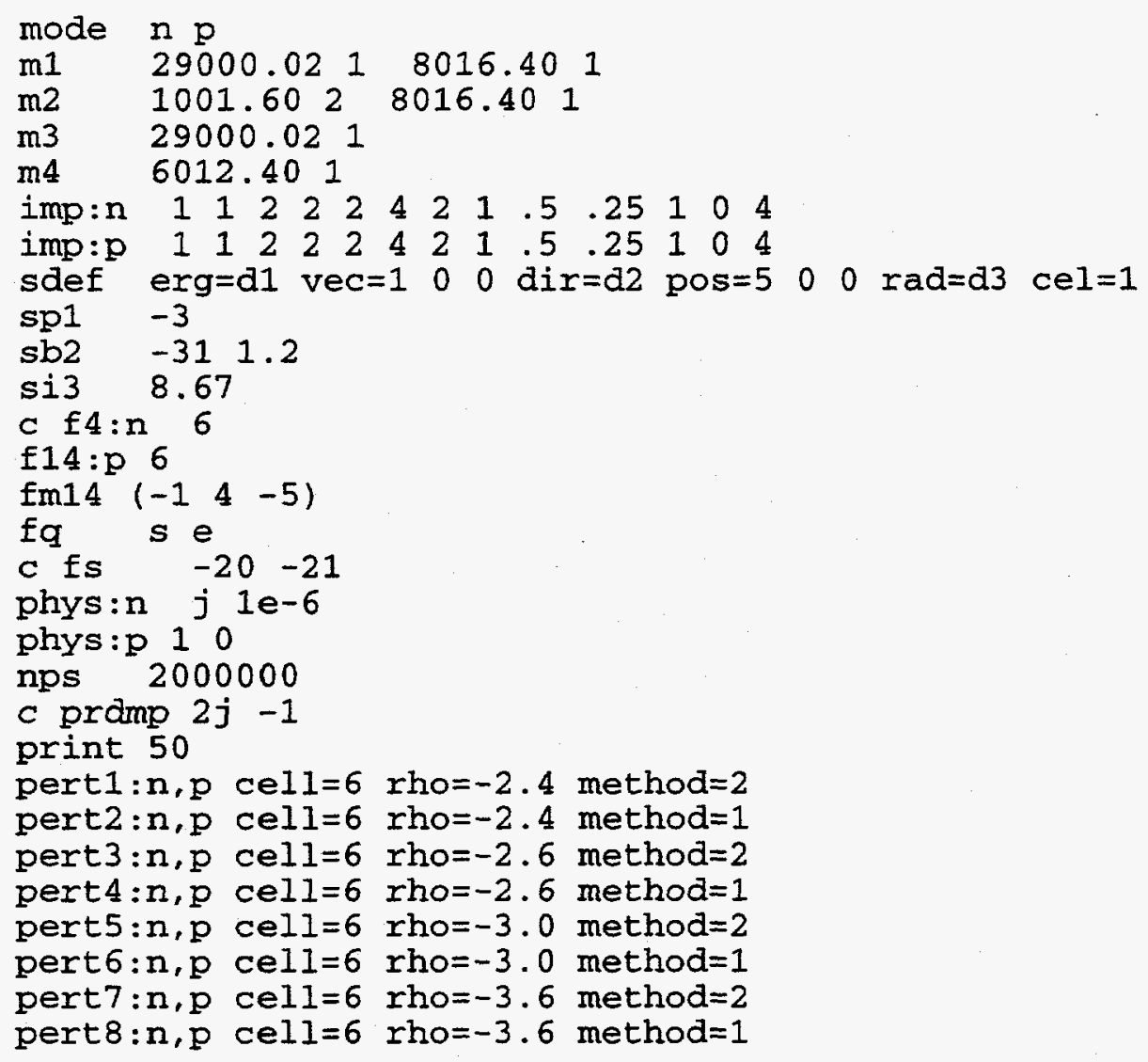

The "true" results for the four perturbations are obtained by deleting the PERT cards and changing the density of cell 6 , material 4 , line 7 ,

$$
\begin{array}{llllll}
6 & 4 & -2.25 & 7 & -8 & -12
\end{array}
$$

with the first perturbation, input=inp102,

6

$$
\begin{array}{lllll}
4 & -2.4 & 7 & -8 & -12
\end{array}
$$

or with the second perturbation, input=inp103,

$$
\begin{array}{lllllll}
6 & 4 & -2.6 & 7 & -8 & -12
\end{array}
$$

or with the third perturbation, input=inp104,

6

$$
\begin{array}{lllll}
4 & -3.0 & 7 & -8 & -12
\end{array}
$$

or with the fourth perturbation, input=inp105,

$$
6
$$

$$
\begin{array}{llllll}
4 & -3.6 & 7 & -8 & -12
\end{array}
$$

